\title{
Optimal priority-free conditionally- preemptive real-time scheduling of periodic tasks based on DES supervisory control
}

\author{
Xi Wang, Zhiwu Li, W.M. Wonham
}

\author{
Version Post-print/accepted manuscript \\ Citation X. Wang; Z. Li; W. M. Wonham, "Optimal Priority-Free Conditionally- \\ (published version) Preemptive Real-Time Scheduling of Periodic Tasks Based on DES \\ Supervisory Control," in IEEE Transactions on Systems, Man, and \\ Cybernetics: Systems, vol.PP, no.99, pp.1-17, 2016. doi: \\ 10.1109/TSMC.2016.2531681
}
Publisher's statement (C) (C) 20xx IEEE. Personal use of this material is permitted. Permission from IEEE must be obtained for all other uses, in any current or future media, including reprinting/republishing this material for advertising or promotional purposes, creating new collective works, for resale or redistribution to servers or lists, or reuse of any copyrighted component of this work in other works.

Copyright/License (C) (C) 20xx IEEE.

How to cite TSpace items

Always cite the published version, so the author(s) will receive recognition through services that track citation counts, e.g. Scopus. If you need to cite the page number of the author manuscript from TSpace because you cannot access the published version, then cite the TSpace version in addition to the published version using the permanent URI (handle) found on the record page.

This article was made openly accessible by $\mathbf{U}$ of $T$ Faculty. Please tell us how this access benefits you. Your story matters. 


\title{
Optimal Priority-Free Conditionally-Preemptive Real-Time Scheduling of Periodic Tasks Based on DES Supervisory Control
}

\author{
Xi Wang, ZhiWu Li, Senior Member, IEEE, and W. M. Wonham, Life Fellow, IEEE
}

\begin{abstract}
This study presents a general discrete event system (DES)-based hard periodic real-time task model. Based on supervisory control theory (SCT), an optimal priority-free real-time scheduling technique is proposed to process all the tasks running in uni-processor or multi-processor real-time systems (RTS). The preemption relation in this paper generalizes priority-based preemption. First, regular languages are utilized to describe the processor behavior related to each task's execution. Thereafter, the languages are represented by DES generators. Finally, the global processor behavior is generated as the synchronous product of these DES generators. A novel preemption policy, namely conditional-preemption, is developed. Two sets of conditionalpreemption specifications are developed, on the processor level and task level, respectively. Moreover, in order to control the system to be nonblocking and also limit the worst-case response time (WCRT) of the tasks, two corresponding sets of specifications are presented. After generating the global specification as the synchronous product, by implementing SCT the calculated supervisor can provide all the safe real-time execution sequences. The supervisor calculation can be sped up by a three step algorithm. Finally, the real-time scheduling is implemented for real-world examples.
\end{abstract}

Index Terms-Real-time system, scheduling, discrete-event system, supervisory control, limited preemptive, conditionalpreemptive. of China under Grant No. 61374068 and the Science and Technology Development Fund, MSAR, under Grant No. 066/2013/A2.

$\mathrm{X}$. Wang is with the School of Electro-Mechanical Engineering, Xidian University, Xi'an 710071, China, and also with the Systems Control Group, Department of Electrical and Computer Engineering, University of Toronto, Toronto, ON M5S 3G4, Canada (e-mail: wanggkingg@gmail.com).

$\mathrm{Z}$. W. Li is with the Institute of Systems Engineering, Macau University of Science and Technology, Taipa, Macau, and also with the School of ElectroMechanical Engineering, Xidian University, Xi'an 710071, China (e-mail: zhwli@xidian.edu.cn).

W. M. Wonham is with Department of Electrical and Computer Engineering, University of Toronto, Toronto, ON M5S 3G4, Canada (e-mail: wonham@control.utoronto.ca). fixed-priority (FP) sequences are included. If the supervisor is empty, the RTS is claimed to be non-schedulable. Otherwise, users can choose any sequence to schedule the RTS.

Compared with non-preemptive scheduling, preemptability can provide more flexibility to real-time scheduling. In fully preemptive systems, at any time, the execution of a running task can be interrupted by tasks with higher priorities, and it continues when all tasks with higher priorities have completed [19].

However, for the real-time scheduling in a processor, both preemptive and non-preemptive scheduling policies are too conservative. Generally, users may customize specific preemption plans that are neither preemptive nor non-preemptive. Recently, several studies are devoted to different preemption policies; these can be divided into two categories:

- Priority-based: Preemption thresholds scheduling (PTS) [20], under FP scheduling, allows a task to disable preemption up to a specified priority level. Each task is assigned a fixed priority and a preemption threshold, and only tasks with priorities higher than the threshold of the running task can preempt its execution.

- Task-based: Deferred preemptions scheduling (DPS) [21], under EDF scheduling, assigns each task a maximum interval that is free from preemption.

The two preemption policies are considered as the tradeoff between preemptive and non-preemptive real-time scheduling. They can significantly improve the EDF and FP real-time scheduling algorithms, respectively. However, both policies are still limited by priorities. In fact, for some real-time scheduling requirements, priorities cannot be assigned to real-time tasks. Moreover, to the best of our knowledge, no existing work can combine the two latter policies together into a real-time scheduling strategy and find all the safe execution sequences. For example, under FP or EDF scheduling, an RTS cannot be scheduled by satisfying PTS and DPS simultaneously.

In this paper, a discrete-event system (DES) real-time task model is presented to schedule the real-time tasks running in uni-processor or multi-processor systems. The preemption relation in this paper generalizes priority-based preemption. Regular languages are utilized to describe the processor behavior related to the execution and preemption of each task. Each language can be represented by a DES generator. The synchronous product [14] of these DES generators can integrate the models of the tasks running in a processor into a complex generator to represent the global processor behavior. For each processor, without considering the priori- 
ties, this present study extends the PTS and DPS scheduling policies to two general conditional-preemption specifications. They are utilized to customize scheduling and preemption requirements conditionally, such as FP, PTS, DPS, preemptive scheduling, and non-preemptive scheduling. Moreover, the worst-case response time (WCRT) of a task can also be restricted by a specification. Thereafter, similar to the work in [13], all the safe execution sequences generated by the synchronized specifications with respect to the tasks running in each processor can be calculated offline by SCT. Users can choose any sequence to schedule the processor. The realtime scheduling with conditional-preemption is applied to realworld uni-processor or multi-processor systems.

The rest of this paper is organized as follows. Section II presents the terminology used throughout the paper. The system model and motivation are described in Section III. The priority-free real-time scheduling with conditional-preemption principles is defined in Section IV. The DES model for the periodic tasks and the RTS are proposed in Section V. The specifications are formalized and established in Section VI. By applying to real-world systems, Section VII reports methodologies for the real-time scheduling with conditionalpreemption. Further relevant issues are discussed in Section VIII. Finally, Section IX states our conclusions.

\section{CONCEPTS AND TERMINOLOGY}

A DES plant is a generator

$$
\mathbf{G}=\left(Q, \Sigma, \delta, q_{0}, Q_{m}\right)
$$

where

- $Q$ is the finite state set,

- $\Sigma$ is the finite event set (alphabet), partitioned into the controllable event subset $\Sigma_{c}$ and the uncontrollable event subset $\Sigma_{u}$;

- $\delta: Y \times \Sigma \rightarrow Y$ is the partial state transition function;

- $q_{0}$ is the initial state; and

- $Q_{m} \subseteq Q$ is the subset of marker states.

In accordance with [14], $\Sigma^{+}$denotes the set of all finite sequences of symbols in $\Sigma$. By adjoining the empty string $\epsilon$, the set of strings over the alphabet $\Sigma$ is written as $\Sigma^{*}$, i.e., $\Sigma^{*}=\Sigma^{+} \cup\{\epsilon\}$. Function $\delta$ can be extended to $\delta: Y \times \Sigma^{*} \rightarrow$ $Y$. We write $\delta(q, s)$ ! to mean that $\delta(q, s)$ is defined, where state $q \in Q$ and string $s \in \Sigma^{*}$. The length $|s|$ of a string $s \in \Sigma^{*}$ is defined as

$$
|\epsilon|=0 ;|s|=k, \text { if } s=\sigma_{1} \sigma_{2} \cdots \sigma_{k} \in \Sigma^{+} .
$$

The closed behavior of $\mathbf{G}$ is represented by

$$
L(\mathbf{G}):=\left\{s \in \Sigma^{*} \mid \delta\left(q_{0}, s\right) !\right\}
$$

and the marked behavior is

$$
L_{m}(\mathbf{G}):=\left\{s \in L(\mathbf{G}) \mid \delta\left(q_{0}, s\right) \in Q_{m}\right\} \subseteq L(\mathbf{G}) .
$$

Synchronous product [14] is a standard way to combine a finite set of DES into a single and more complex one. Suppose that we have $n$ languages corresponding to $n$ DES, $L_{i} \subseteq \Sigma_{i}^{*}$ with $\Sigma=\bigcup_{i \in \mathbf{n}} \Sigma_{i}, \mathbf{n}:=\{1,2, \ldots, n\}$. The natural projection $P_{i}: \Sigma^{*} \rightarrow \Sigma_{i}^{*}$ is defined by
- $P_{i}(\epsilon)=\epsilon$,

- $P_{i}(\sigma)=\left\{\begin{array}{ll}\epsilon, & \text { if } \sigma \notin \Sigma_{i} \\ \sigma, & \text { if } \sigma \in \Sigma_{i}\end{array}\right.$,

- $P_{i}(s \sigma)=P_{i}(s) P_{i}(\sigma), s \in \Sigma^{*}, \sigma \in \Sigma$.

The inverse image function of $P_{i}$ is

$$
P_{i}^{-1}: \operatorname{Pwr}\left(\Sigma_{i}^{*}\right) \rightarrow \operatorname{Pwr}\left(\Sigma^{*}\right) .
$$

For $H \subseteq \Sigma_{i}^{*}$,

$$
P_{i}^{-1}(H):=\left\{s \in \Sigma^{*} \mid P_{i}(s) \in H\right\} .
$$

The synchronous product of $L_{1}, L_{2}, \ldots, L_{n}$ is denoted by $L_{1}\left\|L_{2}\right\| \cdots \| L_{n}$ with

$$
L_{1}\left\|L_{2}\right\| \cdots \| L_{n}:=P_{1}^{-1} L_{1} \cap P_{2}^{-1} L_{2} \cap \cdots \cap P_{n}^{-1} L_{n} .
$$

Suppose that the specification language is represented by $E \subseteq \Sigma^{*}$. Let $\mathcal{C}(E)$ be the family of sublanguages of $E$ that are controllable with respect to $\mathrm{G} . \mathcal{C}(E)$ is nonempty and is closed under arbitrary unions. The (unique) supremal element within $\mathcal{C}(E)$, denoted by $\sup \mathcal{C}(E)$, always exists.

Suppose that a DES model is nonempty. Under supervisory control, all the uncontrollable events are automatically enabled. After adjoining a particular subset of the controllable events to be enabled, a set of all control patterns is defined as

$$
\Gamma=\left\{\gamma \in \operatorname{Pwr}(\Sigma) \mid \gamma \supseteq \Sigma_{u}\right\}
$$

where $\operatorname{Pwr}(\Sigma)$ is the power set of $\Sigma$. A supervisory control for $\mathbf{G}$ is any map $V: L(\mathbf{G}) \rightarrow \Gamma$. DES $\mathbf{G}$ under the supervision of $V$ is written as $V / \mathbf{G}$. The closed behavior of $V / \mathbf{G}$ is defined to be $L(V / \mathbf{G}) \subseteq L(\mathbf{G})$ described as

- empty string $\epsilon \in L(V / \mathbf{G})$,

- if $s \in L(V / \mathbf{G}), \sigma \in V(s)$, and $s \sigma \in L(\mathbf{G})$ then $s \sigma \in$ $L(V / \mathbf{G})$,

- no other strings belong to $L(V / \mathbf{G})$.

The marked behavior of $V / \mathbf{G}$ is

$$
L_{m}(V / \mathbf{G})=L(V / \mathbf{G}) \cap L_{m}(\mathbf{G}) .
$$

The control map $V$ is nonblocking for $\mathbf{G}$ if

$$
\overline{L_{m}(V / \mathbf{G})}=L(V / \mathbf{G}) .
$$

A language $K \in \Sigma^{*}$ is controllable (with respect to $\mathbf{G}$ ) if

$$
\bar{K} \Sigma_{u} \cap L(\mathbf{G}) \subseteq \bar{K}
$$

i.e.,

$$
(\forall s, \sigma) s \in \bar{K} \& \sigma \in \Sigma_{u} \& s \sigma \in L(\mathbf{G}) \Rightarrow s \sigma \in \bar{K} .
$$

Let $K \subseteq L(\mathbf{G})$ be nonempty and closed. There exists a supervisory control $V$ for $\mathbf{G}$ such that $L(V / \mathbf{G})=K$ iff $K$ is controllable with respect to $\mathbf{G}$; this is referred to as a nonblocking supervisory control (NSC). Generally, if marking is also considered, then we select a sublanguage $M \subseteq L_{m}(\mathbf{G})$. A marking nonblocking supervisory control (MNSC) with respect to $\mathbf{G}$ exists, which is a map $V: L(\mathbf{G}) \rightarrow \Gamma$ with the behavior

$$
L_{m}(V / \mathbf{G})=L(V / \mathbf{G}) \cap M .
$$




\section{System Model And Motivation}

In the literature of real-time scheduling, the existing preemption algorithms are defined based on (dynamic or fixed) priorities, which means that when several tasks are simultaneously eligible to be processed, only a task with the highest priority is permitted to enter the processor. In order to relax this constraint and provide all the tasks an equal chance to be processed, an optimal policy, named priorityfree scheduling policy, is presented. This policy allows users to define conditional-preemption requirements freely without assigning task priorities.

\section{A. System Model}

Suppose that an RTS $\mathbb{S}$ processes a set of independent synchronous/asynchronous periodic tasks, i.e., $\mathbb{S}=\left\{\tau_{1}, \tau_{2}, \ldots\right.$, $\left.\tau_{n}\right\}$. For $i \in \mathbf{n}$, task $\tau_{i} \in \mathbb{S}$ is in the form of a four-tuple

$$
\tau_{i}=\left(R_{i}, C_{i}, D_{i}, T_{i}\right)
$$

with

- a release time $R_{i}$,

- a worst-case execution time (WCET) $C_{i}$,

- a hard deadline $D_{i}$, and

- a period $T_{i}$ with $R_{i} \geq 0$ and $C_{i} \leq D_{i} \leq T_{i}$.

Here $R_{i}, C_{i}, D_{i}$, and $T_{i}$ are non-negative integers representing the lengths of processor time units. A synchronous (resp., asynchronous) task $\tau_{i}$ has $R_{i}=0$ (resp., $R_{i}>0$ ). The processor utilization of $\tau_{i}$ is $U_{i}=\frac{C_{i}}{T_{i}}$.

Suppose that an RTS $\mathbb{S}$ is a uni-processor system; and the total processor utilization of $\mathbb{S}$ is $U_{\mathbb{S}}=\sum_{i=1}^{n} U_{i}$. Similarly, suppose that an RTS $\mathbb{S}$ is a multi-processor system that possesses a processor set $\Xi$ that consist of $s$ processors, i.e., $\Xi=\left\{\Xi_{1}, \Xi_{2}, \ldots, \Xi_{s}\right\}$. The processor utilization of $\Xi_{j}$ is the sum of the processor utilization of all the tasks running in it, i.e., $U_{j}^{\Xi}=\sum_{i} U_{i}, \tau_{i} \in \Xi_{j}$. In both uni-processor and multi-processor systems, a processor is not schedulable in case there is overload [1]. The present study allows a task to enter any processor randomly. However, once a task enters a processor, it cannot be shifted to another. In a processor, realtime scheduling policies can be divided into the following two categories:

- preemptive: a running task can be interrupted by the execution of other released tasks;

- non-preemptive: the execution of a running task cannot be interrupted.

\section{B. Motivation}

For some real-world preemption policies, the tasks running in a processor cannot be assigned with priorities. Next we present a motivating example.

Suppose that a uni-processor RTS $\mathbb{S}$ executes four tasks $\tau_{1}$, $\tau_{2}, \tau_{3}$, and $\tau_{4}$. Their parameters are shown in Table I. We assume that the execution of $\tau_{1}$ can be preempted only by $\tau_{2}$, $\tau_{2}$ only by $\tau_{4}$, and $\tau_{4}$ only by $\tau_{1}$, respectively; moreover, $\tau_{3}$ cannot be preempted. No priorities can be assigned to these tasks. In this case, the EDF, FP, and PTS algorithms cannot be utilized to schedule this RTS. In order to solve such problems, we discard the priorities and refer to the real-time scheduling as priority-free.

TABLE I: Parameters of four tasks

\begin{tabular}{lllll}
\hline task & $R$ & $C$ & $D$ & $T$ \\
\hline$\tau_{1}$ & 0 & 3 & 9 & 9 \\
$\tau_{2}$ & 3 & 3 & 6 & 6 \\
$\tau_{3}$ & 0 & 1 & 4 & 5 \\
$\tau_{4}$ & 0 & 2 & 14 & 18 \\
\hline
\end{tabular}

Based on the priority-free real-time scheduling, conditionalpreemption is presented in this study. By a preemption matrix that will be defined later, users can define any preemption relation among all the tasks running in the same processor. WCETbased conditional-preemption specification is presented in this study. Users can use it to designate how long after its execution is started, a task can be preempted by other tasks. Moreover, the WCRT of a task can also be restricted by a specification. Finally, all the safe execution sequences generated by the synchronized specifications with respect to the tasks running in each processor can be calculated offline by SCT. Users can choose any sequence to schedule the processor.

\section{Priority-Free HARd RTS with PERIOdIC TASKS}

Since both uni-processor and multi-processor real-time systems are considered, theoretical analysis in this section is based on processors instead of real-time systems.

\section{A. Priority-Free Real-Time Scheduling}

Definition 1: [priority-free] A scheduling policy is said to be priority-free if all the released tasks in a processor can be processed in any order.

From the perspective of an individual task $\tau_{i}$, in each hyperperiod [22], all the processor time units are partitioned into:

- busy time: the processor is occupied by other tasks, so $\tau_{i}$ cannot be executed;

- running time: $\tau_{i}$ is in process;

- preemption time (if any): after $\tau_{i}$ has started, its execution is interrupted by (a subset of) other tasks; and

- free time: the execution of $\tau_{i}$ is completed or $\tau_{i}$ has not arrived yet. These processor time units can be idle or utilized to execute other tasks.

Priority-free scheduling policy can be utilized to schedule all the periodic tasks randomly, i.e., the busy time and the preemption time of each task can be occupied by other tasks. Moreover, in accordance with traditional real-time scheduling, a task is not allowed to be interrupted if the system would thereby be left idle. In this case, the free time is also allowed to be left idle only when no task in process.

\section{Example.}

For $\tau_{2}$ shown in Table I, a possible conditionally-preemptive real-time scheduling is illustrated in Fig. 1. By allowing preemption of other tasks, the first nine processor time units are partitioned into: 
- busy time: time interval $[3,4]$;

- running time: time intervals $[4,6]$ and $[7,8]$;

- preemption time: time interval $[6,7]$; and

- free time: time intervals $[0,3]$ and $[8,9]$.

The time intervals $[0,4],[6,7]$, and $[8,9]$ can be occupied by other tasks running in the same processor. Moreover, only time intervals $[0,3]$ and $[8,9]$ could alternatively be idle.

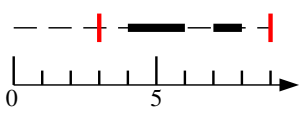

Fig. 1: Real-time scheduling of task $\tau_{2}$.

\section{B. Conditional-Preemption}

From the perspective of processor and individual tasks, two sets of general policies are presented, respectively. They apply to any specific conditional-preemption plans.

\section{1) Matrix-based conditional-preemption:}

In this study, the priority-based preemption policy is extended to a general matrix-based policy. From the perspective of each processor, its preemption matrix is defined to describe the preemption relations among the tasks running in it.

Definition 2: [preemption matrix] An $n \times n$ matrix $P$ is said to be a preemption matrix if $P_{i, j}=1$ (resp., $P_{i, j}=0$ ) represents that a task $\tau_{i}$ is allowed (resp., not allowed) to be preempted by $\tau_{j}$.

Proposition 1: Preemption matrix $P$ can provide up to $2^{n^{2}-n}$ conditionally-preemptive real-time scheduling plans.

Proof: Since $\tau_{i}$ cannot be preempted by itself, we have $P_{i, i}=0$ for all $i \in \mathbf{n}$. If $i \neq j, P_{i, j}$ can be either 0 or 1 . Since there are $n^{2}-n *$ 's in an $n \times n$ matrix $P$, then $P$ can provide up to $2^{n^{2}-n}$ scheduling plans.

\section{Example.}

Suppose that a processor executes all the tasks shown in Table I. Its preemption matrix $P$ is in the form

$$
P=\left(\begin{array}{llll}
0 & * & * & * \\
* & 0 & * & * \\
* & * & 0 & * \\
* & * & * & 0
\end{array}\right)
$$

where $*$, either 0 or 1 , can be predefined by users. According to preemptive real-time scheduling, each task can be preempted by other tasks. Thus, all the $*$ 's in $P$ are replaced by 1 , as shown in $P_{1}$. In contrast, according to non-preemptive realtime scheduling, no task can be preempted by other tasks. Thus, all the $*$ 's in $P$ are replaced by 0 , as shown in $P_{2}$.

Matrix $P_{3}$ describes that

- $\tau_{1}$ can be preempted by $\tau_{2}, \tau_{3}$, and $\tau_{4}$;

- $\tau_{2}$ can be preempted by $\tau_{3}$ and $\tau_{4}$; and

- $\tau_{3}$ can be preempted by $\tau_{4}$.

This is in accordance with the FP real-time scheduling by assigning priorities to tasks $\tau_{1}, \tau_{2}, \tau_{3}$, and $\tau_{4}$ in an increasing order. Similarly, we can assign priorities to tasks $\tau_{1}, \tau_{2}, \tau_{3}$, and $\tau_{4}$ in a decreasing order, as shown in $P_{4}$.
By applying PTS to assign preemption threshold to each task, $P_{3}$ can be revised to be $P_{5}$ as an example. The preemption matrix for the motivating example is $P_{6}$.

$$
\begin{aligned}
& P_{1}=\left(\begin{array}{llll}
0 & 1 & 1 & 1 \\
1 & 0 & 1 & 1 \\
1 & 1 & 0 & 1 \\
1 & 1 & 1 & 0
\end{array}\right) P_{2}=\left(\begin{array}{llll}
0 & 0 & 0 & 0 \\
0 & 0 & 0 & 0 \\
0 & 0 & 0 & 0 \\
0 & 0 & 0 & 0
\end{array}\right) \\
& P_{3}=\left(\begin{array}{llll}
0 & 1 & 1 & 1 \\
0 & 0 & 1 & 1 \\
0 & 0 & 0 & 1 \\
0 & 0 & 0 & 0
\end{array}\right) P_{4}=\left(\begin{array}{llll}
0 & 0 & 0 & 0 \\
1 & 0 & 0 & 0 \\
1 & 1 & 0 & 0 \\
1 & 1 & 1 & 0
\end{array}\right) \\
& P_{5}=\left(\begin{array}{llll}
0 & 0 & 1 & 1 \\
0 & 0 & 0 & 1 \\
0 & 0 & 0 & 1 \\
0 & 0 & 0 & 0
\end{array}\right) P_{6}=\left(\begin{array}{llll}
0 & 1 & 0 & 0 \\
0 & 0 & 0 & 1 \\
0 & 0 & 0 & 0 \\
1 & 0 & 0 & 0
\end{array}\right)
\end{aligned}
$$

321 322 323

Definition 3: [matrix-based conditional-preemption] A preemption policy is said to be matrix-based conditionallypreemptive if it can be represented by a preemption matrix.

2) WCET-based conditional-preemption:

In this study, the task-based preemption policy is extended to a general policy named WCET-based conditionalpreemption. The execution of task $\tau_{i}$ takes $C_{i}$ time units. From the perspective of an individual task, between any two adjacent processor time units the execution of $\tau_{i}$ can be preempted by a subset of other tasks. With respect to task $\tau_{i}$, any two such subsets can be different from each other. Evidently, DPS studied in [21] can be considered as an application of the presented conditional-preemption policy.

In case that WCET-based conditional-preemption relations do not exist, the preemption relations defined in the matrix are applied to the real-time scheduling throughout the execution. Otherwise, the real-time scheduling should take both the WCET-based conditional-preemption and matrixbased conditional-preemption into account simultaneously.

\section{Example.}

According to $P_{1}$ in the last example, $\tau_{2}$ can be preempted by $\tau_{1}, \tau_{3}$, and $\tau_{4}$, which represents that the execution of $\tau_{2}$ can be interrupted by $\tau_{1}, \tau_{3}$, or $\tau_{4}$ immediately upon their arrival. As shown in Table I, we have $C_{2}=3$, i.e., the execution of $\tau_{2}$ takes three time units. Thus we can define two different WCET-based conditional-preemption plans for the execution of $\tau_{2}$, such as 1) between the first two time units, only $\tau_{1}$ and $\tau_{3}$ can interrupt the execution of $\tau_{2}$; and 2) between the last two time units, only $\tau_{1}$ and $\tau_{4}$ can interrupt the execution of $\tau_{2}$.

\section{DES Model for Real-Time Systems}

In this research, regular languages are utilized to describe the processor behavior related to the periodic real-time tasks' execution. Thereafter, each language will be followed by a DES generator representation. Finally, the synchronized language, represented by a more complex DES generator, is utilized to describe the global processor real-time scheduling behavior. In a synchronized DES generator, all the enabled events can occur without considering their priorities. Thus, 
if two or more events are eligible simultaneously, their synchronous product allows them to occur in any order. Since synchronous product can provide all the possible sequences that are not related to priorities, conditional-preemption is possible.

\section{Example.}

Suppose that we have three DES generators $\mathbf{G}_{a}, \mathbf{G}_{b}$, and $\mathbf{G}_{c}$. As depicted in Fig. 2, their alphabets are $\Sigma_{i}=\left\{\sigma_{i}, \sigma_{d}\right\}$, $i \in\{a, b, c\}$. Consequently, $L_{m}\left(\mathbf{G}_{i}\right)=\left(\sigma_{i} \sigma_{d}\right)^{*}$. The synchronous product, $\mathbf{G}_{a} \| \mathbf{G}_{b}$ (resp., $\mathbf{G}_{a}\left\|\mathbf{G}_{b}\right\| \mathbf{G}_{c}$ ), is illustrated in Fig. 3(a) (resp., 3(b)), which has all the 4 (resp., 6) paths, in which $\sigma_{i}$ can occur in any random sequence.

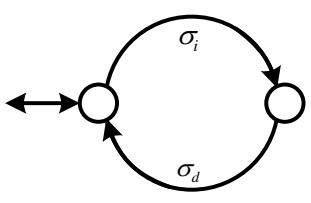

Fig. 2: DES model $\mathbf{G}_{i}$.

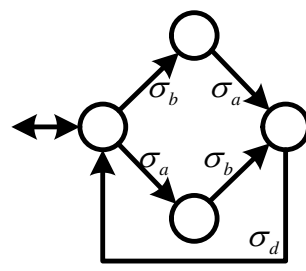

(a) $\mathbf{G}_{a}$ and $\mathbf{G}_{b}$

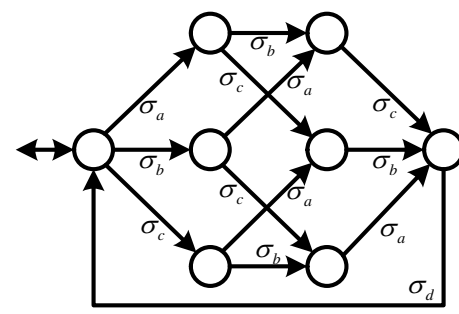

(b) $\mathbf{G}_{a}, \mathbf{G}_{b}$, and $\mathbf{G}_{c}$
Fig. 3: Synchronous product.

\section{A. DES Model for Periodic Tasks}

For any task $\tau_{i} \in \mathbb{S}$ arriving periodically, its execution is represented by a DES generator $\mathbf{G}_{i}$ with marked language $L_{m}\left(\mathbf{G}_{i}\right)$ and (prefix) closed language $L\left(\mathbf{G}_{i}\right)$ satisfying

$$
L\left(\mathbf{G}_{i}\right)=\overline{L_{m}\left(\mathbf{G}_{i}\right)}
$$

that describes all possible executions and random preemptions of task $\tau_{i}$.

\section{1) Regular language representation:}

The alphabet (event labels), written as $\Sigma_{i}$ to describe the behavior of $\mathbf{G}_{i}$, is the disjoint union of $\Sigma_{o i}$ and $\Sigma_{e}$, i.e., $\Sigma_{i}=$ $\Sigma_{o i} \dot{\cup} \Sigma_{e}$, with $\Sigma_{o i} \cap \Sigma_{e}=\emptyset, \Sigma_{o i}=\left\{\gamma_{i}, \alpha_{i}, \beta_{i}\right\}$, and $\Sigma_{e}=$ $\left\{c_{1}, c_{2}, \ldots, c_{i}, \ldots, c_{n}, t\right\}$, where

- $\Sigma_{o i}$ is the operation event set of task $\tau_{i}$, with

- $\gamma_{i}$ : release event,

- $\alpha_{i}$ : the execution of $\tau_{i}$ is started, and

- $\beta_{i}$ : the execution of $\tau_{i}$ is completed;

- $\Sigma_{e}$ is the execution event set, with

- $(i \in \mathbf{n}) c_{i}: \tau_{i}$ is running in the processor, and

- $t$ : no task is running in the processor, i.e., the corresponding processor time unit is idle.

For any $\sigma \in \Sigma_{e}$, the occurrence of $\sigma$ takes a single processor time unit. The marked language $L_{m}\left(\mathbf{G}_{i}\right)$ describes all the possible execution sequences of task $\tau_{i}$ 's execution within a period $T_{i}$. We have

$$
L_{m}\left(\mathbf{G}_{i}\right)=L_{i}^{R}\left(\gamma_{i} L_{i}^{T}\right)^{*} .
$$

This expression contains two parts:

- $L_{i}^{R}$ : the processor behavior before task $\tau_{i}$ is released; and

- $L_{i}^{T}$ : the processor behavior within a period $T_{i}$ between the occurrence of two adjacent $\gamma_{i}$ 's.

The events occurring within $s_{r} \in L_{i}^{R}$ take $R_{i}$ time units in total, i.e.,

$$
L_{i}^{R}=\left\{s_{r}|| s_{r} \mid=R_{i}\right\} .
$$

If $\tau_{i}$ is a synchronous task, then $L_{i}^{R}$ is empty. Otherwise, the set of strings $S_{1} \subseteq\left(\Sigma_{e}-\left\{c_{i}\right\}\right)^{*}$ represents the possible system behavior. Sublanguage $L_{i}^{R}$ could be idle or other tasks being processed earlier than $\gamma_{i}$. For any $\sigma \in \Sigma_{i}$, let $\# \sigma\left(s_{r}\right)$ represent the number of occurrences of $\sigma$ in a string $s_{r}$. We have

$$
L_{i}^{R}=\left\{s_{r} \mid \# t\left(s_{r}\right)+\sum_{j=1, j \neq i}^{n} \# c_{j}\left(s_{r}\right)=R_{i}\right\} .
$$

In a period $T_{i}$, events $\alpha_{i}$ and $\beta_{i}$ must occur only once. Since their occurrences are instantaneous (take no time), and the events occurring within $s \in L_{i}^{T}$ take $T_{i}$ time units in total, the length of every string in a period equals $T_{i}+2$. Formally,

$$
L_{i}^{T}=\left\{s|| s \mid=T_{i}+2\right\} .
$$

For any $s \in L_{i}^{T}$ representing the complete execution of task $\tau_{i}$, the number of $c_{i}$ 's is $C_{i}$, i.e.,

$$
\# c_{i}(s)=C_{i} .
$$

In a period $T_{i}$, i.e., in $L_{i}^{T}$, the processor runs $T_{i}$ processor time units that are occupied by all the execution events. Formally, $s$ satisfies

$$
\# t(s)+\sum_{j=1}^{n} \# c_{j}(s)=T_{i} .
$$

Any string $s \in \gamma_{i} L_{i}^{T}$ contains a substring $s^{\prime}=\gamma_{i} s^{e} \beta_{i}$, in which $s^{e}$ represents the system behavior since the arrival of $\tau_{i}$ until its execution is completed. Thus the response time of task $\tau_{i}$ is the processor time spent between the occurrences of $\gamma_{i}$ and $\beta_{i}$. Since the occurrence of $\alpha_{i}$ is instantaneous, the response time of $\tau_{i}$ in $s$ is

$$
\mathbb{P}_{i}=\left|s^{e}\right|-1 .
$$

The set of strings, $S_{1} \subseteq\left(\Sigma_{e}-\left\{t, c_{i}\right\}\right)^{*}$, occurring earlier than $\alpha_{i}$ is utilized to represent the busy time. Moreover, the preemption time, occurring between $\alpha_{i}$ and $\beta_{i}$ randomly, is represented by a set of strings $S_{2} \subseteq\left(\Sigma_{e}-\left\{t, c_{i}\right\}\right)^{*}$. Furthermore, a set of strings $S_{3} \subseteq\left(\Sigma_{e}-\left\{c_{i}\right\}\right)^{*}$ that occur later than $\beta_{i}$, is utilized to represent the free time. Consequently, for any $s \in L_{i}^{T}, s$ is structurally represented by

$$
s=s_{1} \alpha_{i}\left(s_{2} c_{i} s_{2}\right) \ldots\left(s_{2} c_{i}\right) \beta_{i} s_{3}
$$


with $s_{1} \in S_{1}, s_{2} \in S_{2}$, and $s_{3} \in S_{3}$, respectively. The strings in $\left(\Sigma_{e}-\left\{t, c_{i}\right\}\right)^{*}$ represent the system behavior corresponding to the random preemption by other tasks. String $s_{2}$ occurs between $\alpha_{i}$ and $c_{i}$ or any two adjacent $c_{i}$ 's and represents that the execution of $\tau_{i}$ can be preempted at any time. After the occurrence of the last $c_{i}, \beta_{i}$ occurs immediately to not delay the response time.

Strings in $\left(\Sigma_{e}-\left\{c_{i}\right\}\right)^{*}$ represent the system behavior in the free time. These processor time units can be idle, or utilized to execute other tasks. In order to satisfy the hard deadline $D_{i}$, all the $c_{i}$ 's must occur before $\beta_{i}$. Thus, before the occurrence of $\beta_{i}$, the preemption time cannot be longer than $D_{i}-C_{i}$. Formally, strings $s_{1}$ and $s_{2}$ within Eq. (9) form sublanguages $S_{1}$ and $S_{2}$ that also satisfy Eqs. (10) and (11) as follows:

$$
\begin{aligned}
& S_{1}=\left\{s_{1} \mid 0 \leq \sum_{j=1, j \neq i}^{n} \# c_{j}\left(s_{1}\right) \leq D_{i}-C_{i}\right\} \\
& S_{2}=\left\{s_{2} \mid 0 \leq \sum_{j=1, j \neq i}^{n} \# c_{j}\left(s_{2}\right) \leq D_{i}-C_{i}\right\}
\end{aligned}
$$

By Eq. (7), the free time cannot be longer than $T_{i}-C_{i}$. Formally, sublanguage $S_{3}$ in Eq. (9) must also satisfy

$$
S_{3}=\left\{s_{3} \mid 0 \leq \# t\left(s_{3}\right)+\sum_{j=1, j \neq i}^{n} \# c_{j}\left(s_{3}\right) \leq T_{i}-C_{i}\right\} .
$$

\section{Example.}

The closed and marked languages to describe the processor behavior to execute task $\tau_{1}$ are

$$
L\left(\mathbf{G}_{1}\right)=\overline{L_{m}\left(\mathbf{G}_{1}\right)}
$$

and

$$
L_{m}\left(\mathbf{G}_{1}\right)=L_{1}^{R}\left(\gamma_{1} L_{1}^{T}\right)^{*} .
$$

Sublanguage $L_{1}^{T}$ satisfies $L_{1}^{R}=\epsilon, \forall s \in L_{1}^{T},|s|=8$, $\# c_{1}(s)=2$, and $\# t(s)+\sum_{j=1}^{3} \# c_{j}(s)=6$. String $s$ is structurally represented by

$$
s=s_{1} \alpha_{1} s_{2} c_{1} s_{2} c_{1} \beta_{1} s_{3}
$$

with $s_{1} \in S_{1}, s_{2} \in S_{2}$, and $s_{3} \in S_{3}$ as follows.

$$
\begin{aligned}
& \text { - } S_{1}=\left\{s_{1} \in\left\{c_{2}, c_{3}\right\}^{*} \mid 0 \leq \sum_{j=2}^{3} \# c_{j}\left(s_{1}\right) \leq 4\right\}, \\
& \text { - } S_{2}=\left\{s_{2} \in\left\{c_{2}, c_{3}\right\}^{*} \mid 0 \leq \sum_{j=2}^{3} \# c_{j}\left(s_{2}\right) \leq 4\right\} \text {, and } \\
& \text { - } S_{3}=\left\{s_{3} \in\left\{c_{2}, c_{3}, t\right\}^{*} \mid 0 \leq \# t\left(s_{3}\right)+\sum_{j=2}^{3} \# c_{j}\left(s_{3}\right) \leq 4\right\} .
\end{aligned}
$$

So far, the regular language description of processor behavior related to each task's execution is well defined. The next step is to utilize an appropriate DES model to represent the regular language satisfying Eqs. (1)-(12) simultaneously.

\section{2) DES generator representation:}

The DES generator for task $\tau_{i}$ is represented by

$$
\mathbf{G}_{i}=\left(Q_{i}, \Sigma_{i}, \delta_{i}, q_{0 i}, Q_{m i}\right)
$$

where
- $Q_{i}$ is the finite state set,

- $\Sigma_{i}$ is the alphabet with $\Sigma_{i}=\Sigma_{o i} \dot{\cup} \Sigma_{e}$ it can also be partitioned into $\Sigma_{i}=\Sigma_{c i} \dot{U} \Sigma_{u i}$, with

- $\Sigma_{c i}=\left\{\alpha_{i}, c_{1}, c_{2}, \ldots, c_{i}, \ldots, c_{n}\right\}:$ controllable event subset and

- $\Sigma_{u i}=\left\{\beta_{i}, \gamma_{i}, t\right\}:$ uncontrollable event subset;

- $\delta_{i}: Q_{i} \times \Sigma_{i} \rightarrow Q_{i}$ is the (partial) transition function,

- $q_{0 i}$ is the initial state, and

- $Q_{m i}$ is the subset of marker states.

The controllability of $\alpha_{i}$ (resp. $i \in \mathbf{n}, c_{i}$ ) endows the uniprocessor with the authority to choose and execute (resp. interrupt) any task among all the released ones. Disabling the controllable event $\alpha_{i}$ is utilized to delay the execution of task $\tau_{i}$ for the purpose of avoiding blocking. The general DES model for real-time periodic tasks is presented in Fig. 4. The subscript of each state's name consists of two parts. All the states and transitions are defined by:

- states $y_{0 i, 0}, y_{0 i, 1}, \ldots, y_{0 i, R_{i}-1}$ form the state set before the task releases for the first time;

- state $y_{0 i, R_{i}}: \tau_{i}$ releases;

- for the other states except $y_{0 i,-}$, the first subscript is the past processor time unit after the arrival of task $\tau_{i}$ in the current period, and the second is the corresponding operation related to $\tau_{i}$ or the amount of processor time units already utilized to process $\tau_{i}$. These states are defined as

- $\left(i \in \mathbf{n}, 0 \leq j \leq D_{i}-C_{i}\right) y_{j, \alpha_{i}}$ : the processor is ready to process $\tau_{i}$,

- $\left(i \in \mathbf{n}, C_{i} \leq j \leq D_{i}\right) y_{j, \beta_{i}}$ : the execution of $\tau_{i}$ is complete,

- $\left(0 \leq j \leq T_{i}, 0 \leq k \leq C_{i}\right) y_{j, k}$ : during the first $j$ processor time unit after the release of $\tau_{i}, k$ time units have been utilized to process $\tau_{i}$,

- $y_{T_{i}, \beta_{i}}=y_{0 i, R_{i}}$,

- $y_{T_{i}, \beta_{i}}=y_{0 i, R_{i}}, D_{i}=T_{i} \Rightarrow y_{D_{i}, \beta_{i}}=y_{0 i, R_{i}}$, and

- the states with outgoing arrows are the marker states $Q_{m}$;

- the state with a double arrow is the initial and marker state $y_{0 i, 0}$

- $c_{i}$ (resp., $c_{j}$ ) represents the execution of $\tau_{i}$ (resp., $\tau_{j} \in \mathbb{S}$, $j \neq i$ ); and

- the function $\delta_{i}$ satisfies

- $\left(0 \leq p \leq R_{i}-1\right) \delta\left(y_{0 i, p}, t\right)=y_{0 i, p+1}$ : the processor time units are idle;

- $\left(0 \leq p \leq R_{i}-1\right) \delta\left(y_{0 i, p}, c_{j}\right)=y_{0 i, p+1}: \tau_{j} \in \mathbb{S}$, $j \neq i$ is in process;

- $\delta\left(y_{0 i, R_{i}}, \gamma_{i}\right)=y_{0 i, \alpha_{i}}: \tau_{i}$ is released;

- $\left(0 \leq p \leq D_{i}-C_{i}\right) \delta\left(y_{p, \alpha_{i}}, \alpha_{i}\right)=y_{p, 0}$ : at state $y_{p, \alpha_{i}}$, the execution of $\tau_{i}$ is started;

- $\left(C_{i} \leq p \leq D_{i}\right) \delta\left(y_{p, C_{i}}, \beta_{i}\right)=y_{p, \beta_{i}}$ : at state $y_{p, C_{i}}$, the processing of $\tau_{i}$ is completed;

- $\left(0 \leq p \leq D_{i}-1,0 \leq k \leq C_{i}-1\right) k \leq p$, $\delta\left(y_{p, k}, c_{i}\right)=y_{p+1, k+1}: \tau_{i}$ is in process;

- $\left(0 \leq p \leq T_{i}-1, q \neq c_{i}\right), \delta\left(y_{p, q}, c_{j}\right)=y_{p+1, q}$ : $\tau_{j} \in \mathbb{S}, j \neq i$ is in process;

- $\left(C_{i} \leq p \leq T_{i}-2\right), \delta\left(y_{p, \beta_{i}}, t\right)=y_{p+1, \beta_{i}}$ : after the occurrence of $\beta_{i}$, the processor time units are idle; 


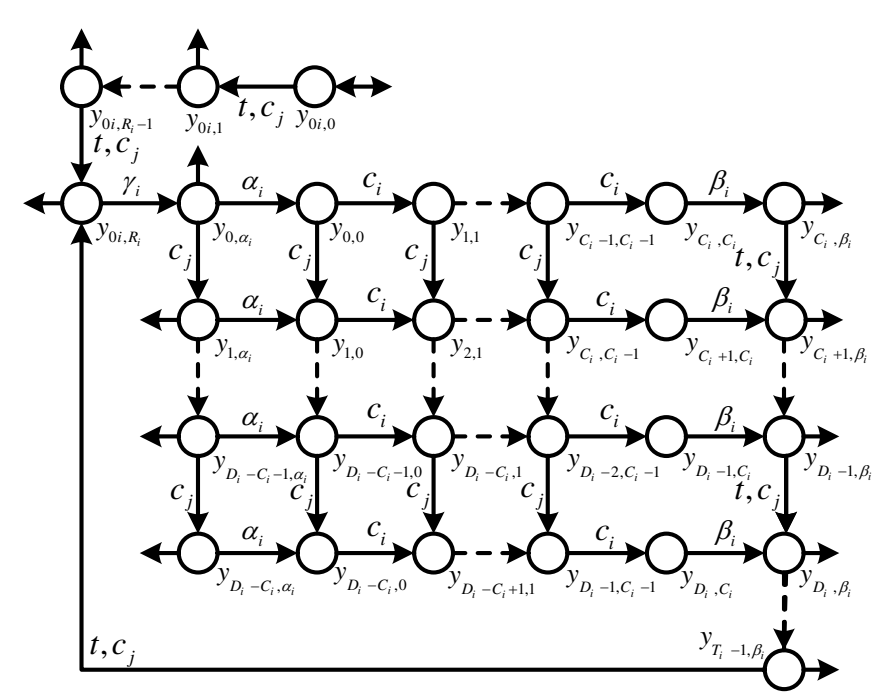

Fig. 4: General DES model for task $\tau_{i}$.

and

- $\delta\left(y_{T_{i}-1, \beta_{i}}, t\right)=y_{0 i}$ : the last processor time unit in $T_{i}$ is idle.

\section{Example.}

The DES model $\mathbf{G}_{2}$ corresponding to tasks $\tau_{2}$ is depicted in Fig. 5. Since $R_{2}=3$, event $\gamma_{2}$ occurs at the end of the third processor time unit. After each period of $\tau_{2}$ is finished, $\gamma_{2}$ occurs immediately to repeat the process.

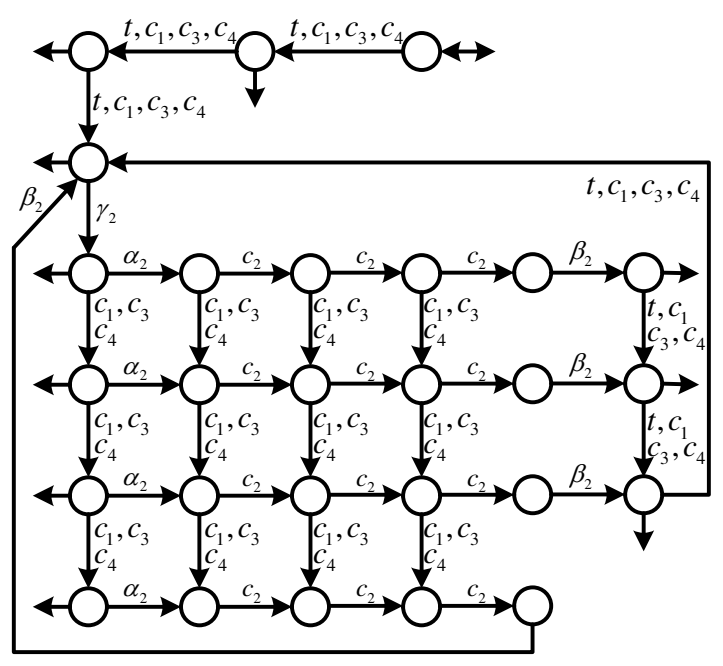

Fig. 5: DES model $\mathbf{G}_{2}$.

\section{B. DES Task Model Creation in TCT}

The software package TCT ${ }^{1}$ is a tool utilized to create the DES generator related to each task. Thereafter, the DES model for RTS is established as the synchronous product [13] of the generated DES models. Further operations to edit the models and/or compute the supervisor can also be executed in TCT. The procedures utilized in this study are summarized in Appendix 1. All the operations in TCT and the names of the

${ }^{1}$ http://www.control.utoronto.ca/DES generated files are recorded in an annotated file MAKEIT.TXT. In TCT, $\mathbf{G}_{1}, \mathbf{G}_{2}, \mathbf{G}_{3}$, and $\mathbf{G}_{4}$ are named as TASK1, TASK2, TASK3, and TASK4, respectively. As an example, TASK2 is reported in Appendix 2. Furthermore, for $i \in \mathbf{n}$, events $\gamma_{i}, \alpha_{i}$, and $\beta_{i}$ are renamed $i 0, i 1$, and $i 2$, respectively. Events $c_{i}$ and $t$ are represented by $i 9$ and 0 , respectively. The system behavior corresponding to each processor is calculated by synchronous product. Suppose that we have an RTS $\mathbb{S}$ that possesses only one processor; three examples are discussed below.

1) Plan 1: $\tau_{1}$ and $\tau_{2}$ in process:

Suppose that tasks $\tau_{1}$ and $\tau_{2}$ are processed in $\mathbb{S}$, denoted by $\mathbb{S}^{1}$. It is generated by the procedure sync provided by TCT. Since tasks $\tau_{3}, \tau_{4} \notin \mathbb{S}^{1}$, within $\tau_{1}$ and $\tau_{2}$, we can eliminate $c_{3}$ and $c_{4}$ (events 39 and 49 ) by relabeling them to be $c_{2}$ (event 29) and $c_{1}$ (event 19), respectively. Thereafter, the DES model representing $\mathbb{S}^{1}$ can be generated by synchronous product. In TCT, $\mathbb{S}^{1}$ is represented by SYS1 that contains 71 states and 98 transitions. The corresponding TCT procedures are:

TEST1 $=$ relabel $($ TASK $1,[[39,29],[49,29]])(42,72)$ TEST2 = relabel $($ TASK2, $[[39,19],[49,19]])(28,45)$ SYS1 = sync $\left(\right.$ TEST1, TEST2) $(71,98)^{2}$

2) Plan 2: $\tau_{1}, \tau_{2}$, and $\tau_{4}$ in process:

Suppose that tasks $\tau_{1}, \tau_{2}$, and $\tau_{4}$ are processed in RTS $\mathbb{S}$, denoted by $\mathbb{S}^{2}$. Named to be SYS2 in TCT, it can be generated in a similar way, i.e.,

$$
\begin{aligned}
& \text { TEST } 1=\text { relabel }(\text { TASK } 1,[[39,29]])(42,102) \\
& \text { TEST2 }=\text { relabel }(\text { TASK2, }[[39,19]])(28,63) \\
& \text { TEST } 4=\text { relabel }(\text { TASK4, [[39, 19]] })(69,173) \\
& \text { SYS2 }=\text { sync }(\text { TEST } 1, \text { TEST2, TEST4 })(170,279)
\end{aligned}
$$

3) Plan 3: $\tau_{1}, \tau_{2}, \tau_{3}$, and $\tau_{4}$ in process:

Suppose that all four tasks $\tau_{1}, \tau_{2}, \tau_{3}$, and $\tau_{4}$ are processed in RTS $\mathbb{S}$, denoted by $\mathbb{S}^{3}$. Represented by SYS3 in TCT, $\mathbb{S}^{3}$ can be generated in a similar way, i.e.,

SYS3 = sync (TASK1, TASK2, TASK3, TASK4) (952, 2056) Blocked_events $=[0]$

There is a blocked event $0(t)$ in SYS3 $\left(\mathbb{S}^{3}\right)$, which represents that in the real-time scheduling, there is no idle time unit. This means that the processor utilization of $\mathbb{S}^{3}$ is $U^{3} \geq 1$. According to the study in [3], $\mathbb{S}^{3}$ is non-schedulable if $U^{3}>1$. By calculating the processor utilization of $\mathbb{S}^{3}$, we obtain $U^{3}=3 / 9+3 / 6+1 / 5+2 / 14>1$. Thus, $\mathbb{S}^{3}$ is nonschedulable. In the modeling phase, "bad decisions" made by synchronous product may block the priority-free conditionallypreemptive real-time scheduling. As a solution, in the rest of this paper, SCT is utilized to supervise the RTS to be nonblocking.

\section{Specifications For Optimal Priority-Free Conditionally-PreEmptive REAL-Time Scheduling}

In accordance with [14], all possible behaviors in a processor are generated by a DES, called the plant. A processor under control is a subset of the generated languages with respect

\footnotetext{
${ }^{2}$ All the original message "Blocked events $=$ None" in the original MAKEIT file are eliminated for readability.
} 
to certain constraints that are contained in some specification languages. In order to schedule the processor to be nonblocking and conditionally-preemptive, we shall impose the synchronous product of proper specifications on the behavior of the processor. For each task running in a processor, four types of specifications are defined:

- Nonblocking specifications: nonblocking preemptive scheduling of real-time tasks;

- Matrix-based conditional-preemption specifications: the preemption relation among all the tasks;

- WCET-based conditional-preemption specifications: during the execution of each task, the exact preemption plan between two adjacent time units; and

- WCRT-based conditional-preemption specifications: the WCRT in all the periods.

\section{A. Nonblocking Specifications}

In order to control the RTS to be nonblocking, the specification $\mathbf{S}_{i}^{N}$ for $\tau_{i}$ should allow the occurrence of any $s \in \Sigma_{i}^{*}$, i.e., $L\left(\mathbf{S}_{i}^{N}\right)=\Sigma_{i}^{*}$. The procedure allevents can be utilized to generate a DES representing $\Sigma_{i}^{*}$. As shown in Fig. 6, the nonblocking specification for task $\tau_{i}$ is a generator with only one state at which all $\sigma \in \Sigma_{i}$ are enabled.

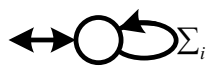

Fig. 6: Nonblocking specification for task $\tau_{i}$.

\section{B. Matrix-Based Conditional-Preemption Specifications}

In a processor, all the possible preemptions that may occur during the execution of $\tau_{i}$ are defined in the $i$-th row of the preemption matrix $P$. More precisely, task $\tau_{i}$ can be preempted by $\tau_{j}$ in case $P_{i, j}=1$. The preemption occurs between the occurrence of $\alpha_{i}$ and $\beta_{i}$. Thus, a specification $\mathbf{S}_{i}^{P}$ is defined for each task $\tau_{i}$ with a generator

$$
\mathbf{S}_{i}^{P}=\left(Q_{i}^{P}, \Sigma_{i}^{P}, \delta_{i}^{P}, q_{0 i}^{P}, Q_{m i}^{P}\right) .
$$

Here

- $Q_{i}^{P}$ : the state set contains two states:

- $y_{0}: \tau_{i}$ is not in process; and

- $y_{1}: \tau_{i}$ is in process;

- $\Sigma_{i}^{P}=\bigcup_{i \in \mathbf{n}} \Sigma_{i}$ : the set of all the events appearing in the processor;

- $\delta_{i}^{P}$ : the (partial) transition function:

- $\delta_{i}^{P}\left(y_{0}, \sigma\right)=y_{0}, \sigma \in \Sigma_{e}-\left\{c_{i}\right\}: \tau_{i}$ is not in process, the time unit can be taken by other tasks or idle;

- $\delta_{i}^{P}\left(y_{1}, c_{i}\right)=y_{1}: \tau_{i}$ is in process;

- $\delta_{i}^{P}\left(y_{1}, c_{j}\right)=y_{1}: \tau_{i}$ can be preempted by $\tau_{j}$, i.e., $P_{i, j}=1$;

- $\delta_{i}^{P}\left(y_{0}, \alpha_{i}\right)=y_{1}$ : the execution of $\tau_{i}$ is started; and

- $\delta_{i}^{P}\left(y_{1}, \beta_{i}\right)=y_{0}$ : the execution of $\tau_{i}$ is completed;

- $Q_{m i}^{P}=\left\{q_{0 i}^{P}\right\}=\left\{y_{0}\right\}$ is the initial and marker state.

The DES model of specification $\mathbf{S}_{i}^{P}$ for $\tau_{i}$ is illustrated in Fig. 7 , where $c_{j}$ represents the execution of task $\tau_{j}$ that is allowed to preempt the execution of $\tau_{i}$.

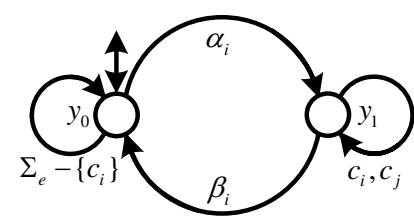

Fig. 7: Matrix-based conditional-preemption specification.

\section{WCET-Based Conditional-Preemption Specifications}

In this section, we define the exact preemption plans between two adjacent time units $\left(c_{i}\right.$ 's) of task $\tau_{i}$. A specification $\mathbf{S}_{i}^{C}$ is defined for each task $\tau_{i}$ with a generator

$$
\mathbf{S}_{i}^{C}=\left(Q_{i}^{C}, \Sigma_{i}^{C}, \delta_{i}^{C}, q_{0 i}^{C}, Q_{m i}^{C}\right) .
$$

Here

- $Q_{i}^{C}$ : the state set containing $C_{i}+2$ states;

- $\Sigma_{i}^{C}=\bigcup_{i \in \mathbf{n}} \Sigma_{i}$ : the set of all the events appearing in the processor;

- $\delta_{i}^{C}$ : the (partial) transition function:

- $\delta_{i}^{C}\left(0_{i}, \sigma\right)=0_{i}, \sigma \in \Sigma_{e}-\left\{c_{i}\right\}: \tau_{i}$ is not in process; and the time unit can be occupied by other tasks or idle;

- $\delta_{i}^{C}\left(0_{i}, \alpha_{i}\right)=y_{0}: \tau_{i}$ is arrived;

- $\delta_{i}^{C}\left(y_{i}, c_{i}\right)=y_{i+1}: \tau_{i}$ is in process;

- $\delta_{i}^{C}\left(y_{i}, c_{j}\right)=y_{i}: \tau_{i}$ is preempted by the execution of $\tau_{j}$;

- $\delta_{i}^{C}\left(y_{C_{i}+1}, \beta_{i}\right)=0_{i}$ : the execution of $\tau_{i}$ is completed; - $Q_{m i}^{C}=\left\{q_{0 i}^{C}\right\}=\left\{0_{i}\right\}$ is the initial and marker state.

The DES model of specification $\mathbf{S}_{i}^{C}$ for $\tau_{i}$ is shown in Fig. 8.

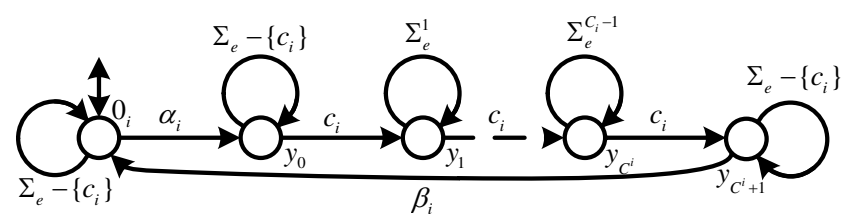

Fig. 8: WCET-based conditional-preemption specification.

\section{WCRT-Based Conditional-Preemption Specifications}

The preemption of real-time execution increases the response time of task $\tau_{i}$. In order to constrain that the execution time of task $\tau_{i}$ to be no longer than a value WCRT $\mathbb{W}_{i}$, i.e., in a period $T_{i}$, the execution between $\gamma_{i}$ and $\beta_{i}$ is limited to be not greater than $\mathbb{W}_{i}$ time units. A specification $\mathbf{S}_{i}^{\mathbb{W}}$ is defined for each task $\tau_{i}$ with a generator

$$
\mathbf{S}_{i}^{\mathbb{W}}=\left(Q_{i}, \Sigma_{i}, \delta_{i}, q_{0 i}, Q_{m i}\right) .
$$

Here

- $Q_{i}^{\mathbb{W}}$ : the state set containing $\mathbb{W}_{i}+2$ states;

- $\Sigma_{i}^{\mathbb{W}}=\Sigma_{i}-\{t\}$ : the set of all the events except $t$ appearing in $\mathbf{G}_{i}$;

- $\delta_{i}^{\mathbb{W}}$ : the (partial) transition function:

- $\delta_{i}^{\mathbb{W}}\left(0_{i}, \sigma\right)=0_{i}, \sigma \in \Sigma_{e}-\left\{c_{i}\right\}: \tau_{i}$ is not in process; and the time unit can be taken by other tasks or idle;

- $\delta_{i}^{\mathbb{W}}\left(0_{i}, \gamma_{i}\right)=y_{0}: \tau_{i}$ is arrived; 
- $\left(c_{j} \in \Sigma_{e}\right) \delta\left(y_{i}, c_{j}\right)=y_{i+1}: \tau_{j}$ is in process;

- $(1 \leq j \leq \mathbb{W}+1) \delta_{i}^{\mathbb{W}}\left(y_{j}, \beta_{i}\right)=0_{i}$ : the execution of $\tau_{i}$ is completed;

- $Q_{m i}^{\mathbb{W}}=\left\{q_{0 i}^{\mathbb{W}}\right\}=\left\{0_{i}\right\}$ : the initial and marker state.

The DES model of specification $\mathbf{S}_{i}^{\mathbb{W}}$ for $\tau_{i}$ is shown in Fig. 9. By Theorem 1 (Theorem 3.5.2 in [14]), $K$ can be found by the procedure supcon (see Appendix 1).

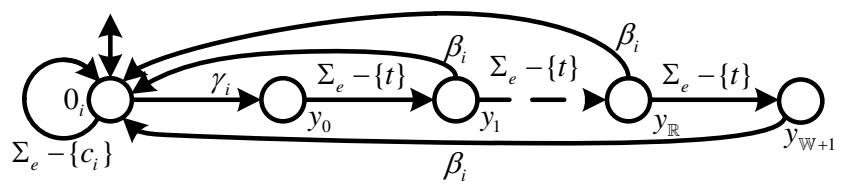

Fig. 9: WCRT-based conditional-preemption specification.

Theorem 1: [14] Let $E \subseteq \Sigma^{*}$ and let $K=\operatorname{supC}(E \cap$ $\left.L_{m}(\mathbf{G})\right)$. If $K \neq \emptyset$ there exists a marking nonblocking supervisory control (MNSC) for $\mathbf{G}$ such that $L_{m}(V / \mathbf{G})=K$.

\section{E. Specification Creation in TCT}

The corresponding TCT operations to create specifications are listed in Appendix 3. In TCT, SN1, SN2, SN3, and SN4 are the nonblocking specifications $\mathbf{S}_{1}^{N}, \mathbf{S}_{2}^{N}, \mathbf{S}_{3}^{N}$, and $\mathbf{S}_{4}^{N}$, respectively. SP1 (resp. SP2) is the non-preemptive specification $\mathbf{S}_{1}^{P}$ (resp. $\mathbf{S}_{2}^{P}$ ) for task $\tau_{1}$ (resp. $\tau_{2}$ ).

The matrix-based conditional-preemptions are created for the PTS example $\left(P_{5}\right)$ and motivating example $\left(P_{6}\right)$.

PTS example $\left(P_{5}\right)$ :

SP134: $\tau_{1}$ can be preempted by $\tau_{3}$ and $\tau_{4}$; and

SP24: $\tau_{2}$ can be preempted by $\tau_{4}$.

Motivating example $\left(P_{6}\right)$ :

SP12: $\tau_{1}$ can be preempted by $\tau_{2}$;

SP24: $\tau_{2}$ can be preempted by $\tau_{4}$; and

SP41: $\tau_{4}$ can be preempted by $\tau_{1}$.

WCET-based conditional-preemption specifications:

SC1: only the last time unit of $\tau_{1}$ can be preempted by $\tau_{2}$;

SC2: only the first time unit of $\tau_{2}$ can be preempted by $\tau_{1}$;

SC3:only the last time unit of $\tau_{1}$ can be preempted by $\tau_{4}$;

SC4: only the first time unit of $\tau_{2}$ can be preempted by $\tau_{4}$.

WCRT-based conditional-preemption specifications:

SR1: the WCRT of task $\tau_{1}$ is $\mathbb{W}_{1}=4$; and

SR4: the WCRT of task $\tau_{4}$ is $\mathbb{W}_{4}=2$.

\section{SUPERVISOR SYNTHESIS}

So far, the priority-free scheduling policy with conditionalpreemption can be described in regular language that can be represented by DES. It is well known that SCT can be used to find the supremal controllers that provide the minimally restricted controller of the systems. TCT is based on SCT, a well-recognized theory in DES modeling and control. By utilizing the procedure sync in TCT, all the specifications can be integrated into a unique one. The procedure supcon in TCT finds all the safe execution sequences within an RTS satisfying the synchronized specification. An offline technique is developed in Algorithm 1 to achieve this goal. Users need not be concerned with the mathematical calculations; and by utilizing TCT, all the safe execution sequences can be provided in the supervisor. Each sequence can be utilized by users to schedule the RTS. All the EDF, FP, and other sequences can be found in the supervisor (Super).

\begin{tabular}{l}
\hline Algorithm 1 Supervisory control of RTS \\
\hline 1. Build Plant by sync; \\
2. Build Spec by sync; \\
3. Super = supcon (Plant, Spec). \\
\hline
\end{tabular}

By comparison, under EDF real-time scheduling the tasks with the earliest deadlines are assigned with the highest priority. Moreover, EDF scheduling chooses only one task among them to execute without considering other possibilities. Other released tasks have no chance to be executed by the processor.

\section{A. Example. 1.}

In this study, as illustrated in Fig. 10, an example motor network similar to the one studied in [13] is considered as an RTS. Suppose that four electric motors are controlled by a uni-processor. Their deadlines and periods are represented by $\mathrm{D}$ and $\mathrm{T}$, respectively. These parameters coincide with those of the tasks in the previous examples as

- Motor 1: $\tau_{1}$,

- Motor 2: $\tau_{2}$,

- Motor 3: $\tau_{3}$, and

- Motor 3: $\tau_{4}$.

The work plans of the motor network also coincide with the RTS models presented in Section V. Since $R_{2}=3$, Motor 2 will be ready three ms later than other tasks. We take the three work plans of $\mathbb{S}$, i.e., $\mathbb{S}^{1}, \mathbb{S}^{2}$, and $\mathbb{S}^{3}$, to find all the safe execution sequences under priority-free conditionallypreemptive scheduling.

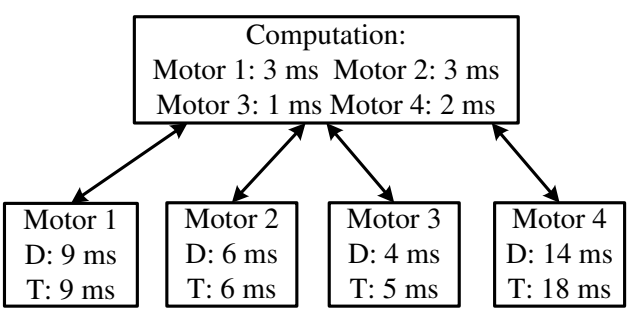

Fig. 10: A motor network example.

\section{B. Supervisory Control of $\mathbb{S}^{1}$}

Since $\tau_{1}, \tau_{2} \in \mathbb{S}^{1}$, we only consider the specifications corresponding to tasks $\tau_{1}$ and $\tau_{2}$. The scheduling corresponding to $P_{1}$ is preemptive. Thus, we only need to control the RTS to be nonblocking. Denote this specification by PS1. According to Algorithm 1, in TCT, it is calculated by

$\mathrm{PS} 1=\operatorname{sync}(\mathrm{SN} 1, \mathrm{SN} 2)(1,11)$

All the safe preemptive execution sequences are calculated by the procedure supcon, i.e., 
PSUP1 = supcon $($ SYS1, PS1) $(71,98)$

The marker states in PSUP1 represent that the corresponding task is ready to be released, which is redundant information for the users. Thus they are unmarked as follows.

PSUP1 = edit $($ PSUP1, [mark -[all]] $)(71,98)$

The safe execution sequence set in PSUP1 is represented by a DES with 84 states and 130 transitions. By projecting out all events but $\alpha_{i}$, i.e.,

PMPSUP1 = project $($ PSUP1, Image $[11,21])(6,7)$

we can obtain the preemptive release map of $\mathbb{S}^{1}$. All the safe release sequences are shown in Fig. 11. In PSUP1, by projecting out all events but $c_{i}$, i.e.,

\section{PJPSUP1 = project $($ PSUP1, Image $[19,29])(24,33)$}

we can obtain the preemptive scheduling map of $\mathbb{S}^{1}$, as shown in Fig. 12(a), that contains all the safe execution sequences of $c_{1}$ and $c_{2}$. The computed supervisor is shown in Table II, in which RM and SM represent the release map and the scheduling map, respectively. The numbers of the states and transitions are recorded in the form (number of states, number of transitions). By Algorithm 1, several other examples by considering different specifications are also listed in Table II. All the release maps are isomorphic with the release map depicted in Fig. 11. Moreover, all the scheduling maps are depicted in Fig. 12. This means that, based on a supremal release map, according to different preemption plans, the priorityfree conditionally-preemptive scheduling policy can provide different results. To the best of our knowledge, no other scheduling algorithms can schedule an RTS by considering $P_{3}$ and SC1 simultaneously.

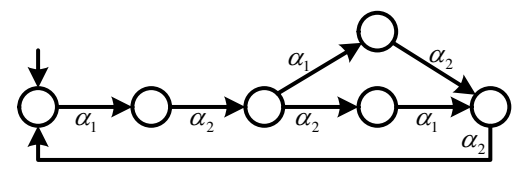

Fig. 11: Release map of $\mathbb{S}^{1}$

TABLE II: Uni-processor scheduler behaviors of $\mathbb{S}^{1}$

\begin{tabular}{llll}
\hline Spec & Super & RM & SM \\
\hline$P_{1}$ & $(71,98)$ & $(6,7)$ & $(24,33)$ \\
$P_{2}$ & $(58,70)$ & $(6,7)$ & $(20,21)$ \\
$P_{3}$ & $(69,87)$ & $(6,7)$ & $(24,27)$ \\
$P_{4}$ & $(69,87)$ & $(6,7)$ & $(24,27)$ \\
$P_{1}$, SC1, SC2 & $(68,90)$ & $(6,7)$ & $(23,27)$ \\
$P_{3}$, SC1 & $(65,82)$ & $(6,7)$ & $(22,24)$ \\
\hline
\end{tabular}

For comparison, the preemptive EDF scheduling result of $\mathbb{S}^{1}$ by Cheddar [23] is depicted in Fig. 13, which can also be found in Fig. 12(a). The execution sequence in Fig. 13 seems like a non-preemptive scheduling sequence. Evidently, none of the exact preemptive scheduling sequences in Fig. 12(a) can be generated by EDF. In case that at $t=9$ in Fig. $13, \tau_{2}$ (with the earliest deadline) cannot arrive on time, and then according to the multiple sequences, users can choose another available sequence shown in Fig. 12(a) to schedule task $\tau_{1}$ first. Thus, recalculating the scheduling sequences is unnecessary. However, at $t=9$, there is no EDF sequence to execute $\tau_{1}$ first. If $\tau_{2}$ cannot arrive on time, the EDF scheduling cannot schedule $\mathbb{S}^{1}$ successfully. The supervisory control technique provides a larger number of safe execution sequences as compared with EDF scheduling. The WCRT of $\tau_{1}$ in the scheduling sequence shown in Fig. 13 is $\mathbb{W}_{1}=6$. By comparison, in the scheduling map shown in Fig. 12(a), when tasks $\tau_{1}$ and $\tau_{2}$ are released simultaneously, they can preempt another randomly. In all these sequences, we have $\mathbb{W}_{1} \leq 6$.

\section{Supervisory Control of $\mathbb{S}^{2}$}

Since $\tau_{1}, \tau_{2}, \tau_{4} \in \mathbb{S}^{1}$, we only consider the specifications corresponding to tasks $\tau_{1}, \tau_{2}$, and $\tau_{4}$. According to Algorithm 1, several supervisors are calculated and listed in Table III. The release maps are isomorphic with each other, as shown in Fig. 14. The scheduling maps of $\mathbb{S}^{2}$ are depicted in Fig. 15. To the best of our knowledge, no sequence in Figs. 15(b) and 15 (d) can be achieved by other scheduling algorithms.

TABLE III: Uni-processor scheduler behaviors of $\mathbb{S}^{2}$

\begin{tabular}{llll}
\hline Spec & Super & RM & SM \\
\hline$P_{5}$ & $(128,186)$ & $(9,12)$ & $(34,41)$ \\
$P_{5}$, SC3, SC4 & $(119,173)$ & $(9,12)$ & $(32,37)$ \\
$P_{6}$ & $(145,21)$ & $(9,12)$ & $(44,54)$ \\
$P_{6}$, SC1 & $(140,204)$ & $(9,12)$ & $(39,47)$ \\
\hline
\end{tabular}

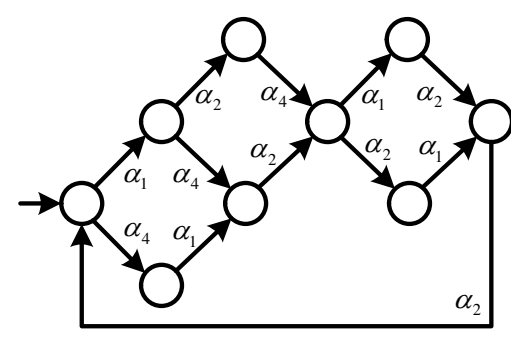

Fig. 14: Release map of $\mathbb{S}^{2}$.

For comparison, the PTS scheduling result of $\mathbb{S}^{2}$ is depicted in Fig. 16, and it can also be found in Fig. 15(a). Evidently, SCT provides a greater number of safe execution sequences as compared with PTS scheduling. The WCRT of $\tau_{4}$ in the scheduling sequence shown in Fig. 16 is $\mathbb{W}_{4}=2$. In comparison, in all the sequences shown in Fig. 15(a), the WCRT of $\tau_{4}$ varies from two to eight. This means that the WCRT in the conditional-preemption scheduling may increase. In this case, by also considering SR4 as a specification, we obtain a supervisor with 83 states and 118 transitions. The corresponding release map is isomorphic with the one shown in Fig. 14. Two scheduling sequences are depicted in Fig. 17; they form a subset of the safe executions shown in Fig. 15(a). The sequence in Fig. 16 can be found in Fig. 17. 


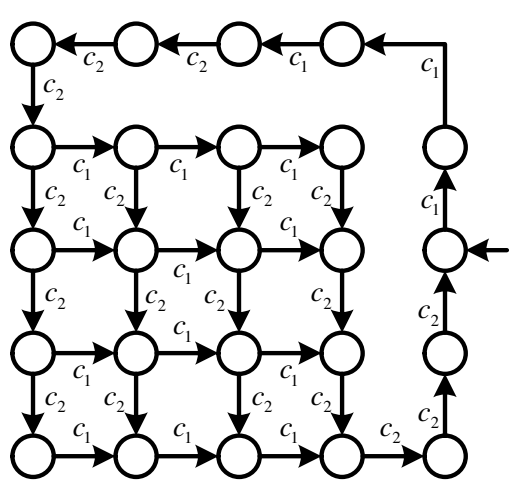

(a) $P_{1}$

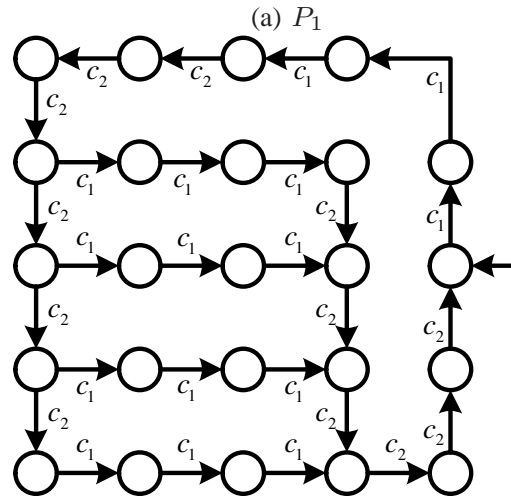

(d) $P_{4}$

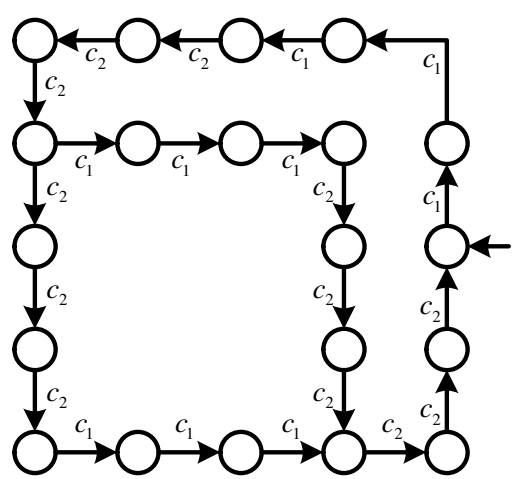

(b) $P_{2}$

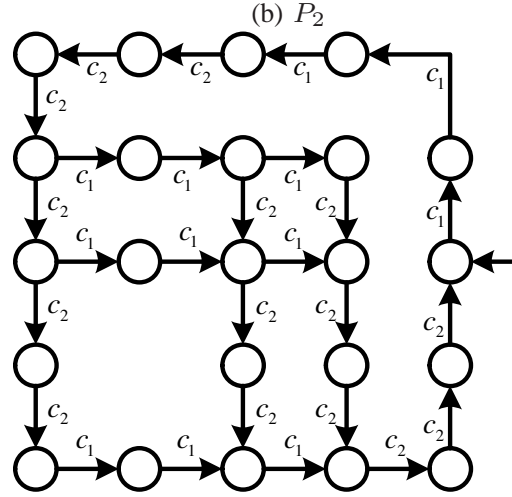

(e) $P_{1}, \mathrm{SC} 1$, and $\mathrm{SC} 2$

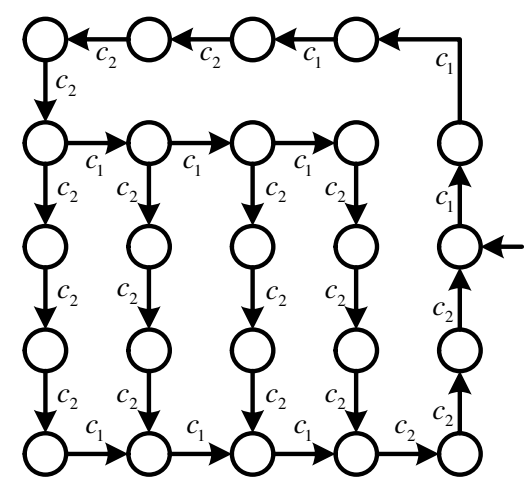

(c) $P_{3}$

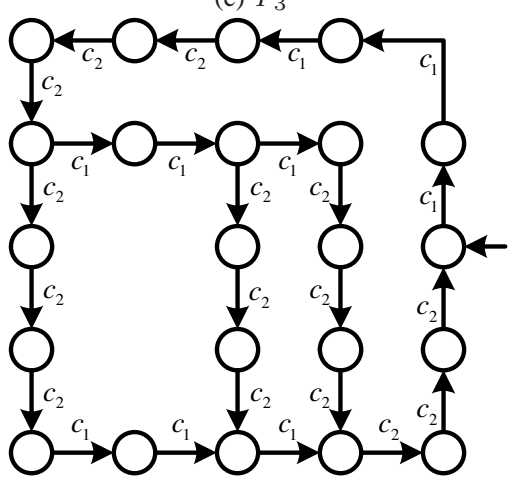

(f) $P_{3}$ and $\mathrm{SC} 1$

Fig. 12: Scheduling map of $\mathbb{S}^{1}$.

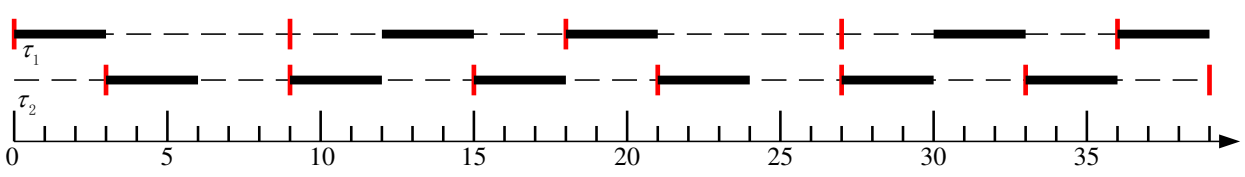

Fig. 13: Preemptive scheduling map of $\mathbb{S}^{1}$.

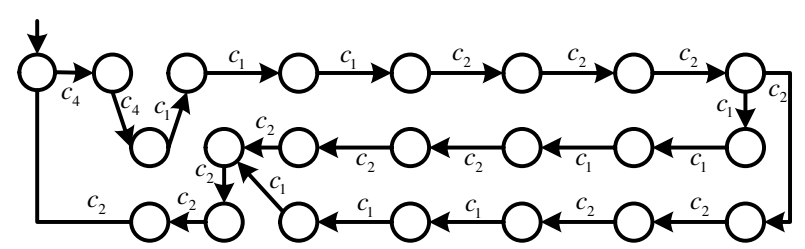

Fig. 17: PTS and WCRT scheduling map of $\mathbb{S}^{2}$.

\section{Example. 2.}

Suppose that we have another motor network, denoted by $\mathbb{S}^{4}$ that executes the five tasks listed in Table IV. Suppose that in a uni-processor system, the preemption matrix takes the following form

$$
P=\left(\begin{array}{ccccc}
0 & * & * & * & * \\
* & 0 & * & * & * \\
* & * & 0 & * & * \\
* & * & * & 0 & * \\
* & * & * & * & 0
\end{array}\right)
$$

which can provide $2^{20}$ matrix-based conditionally-preemptive real-time scheduling plans. It is unnecessary to calculate all the scheduling plans simultaneously. However, each of them can be represented by specifications and the corresponding supervisors can be calculated by SCT.

TABLE IV: Parameters of five tasks

\begin{tabular}{lllll}
\hline task & $R$ & $C$ & $D$ & $T$ \\
\hline$\tau_{1}$ & 0 & 2 & 9 & 9 \\
$\tau_{2}$ & 0 & 2 & 10 & 10 \\
$\tau_{3}$ & 0 & 2 & 12 & 12 \\
$\tau_{4}$ & 0 & 2 & 14 & 16 \\
$\tau_{5}$ & 3 & 2 & 16 & 18 \\
\hline
\end{tabular}

\section{1) Uni-processor system:}

In a uni-processor system, the RTS processing these tasks is represented by a DES generator with 15572 states and 31333 events. Instead of calculating the supervisors of all the $2^{20}$ plans, we arbitrarily choose the following eight plans to calculate the corresponding supervisors. The corresponding supremal supervisors, release maps, and scheduling maps are recorded in Table $\mathrm{V}$.
842 843 


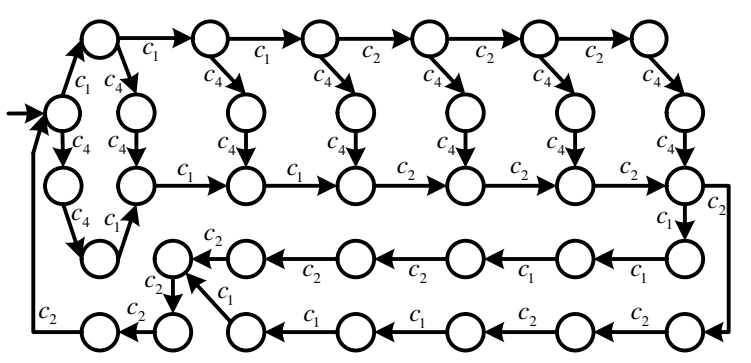

(a) $P_{5}$

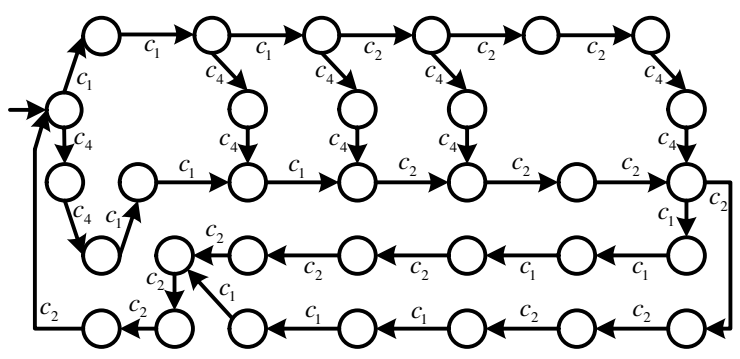

(b) $P_{5}, \mathrm{SC} 3, \mathrm{SC} 4$

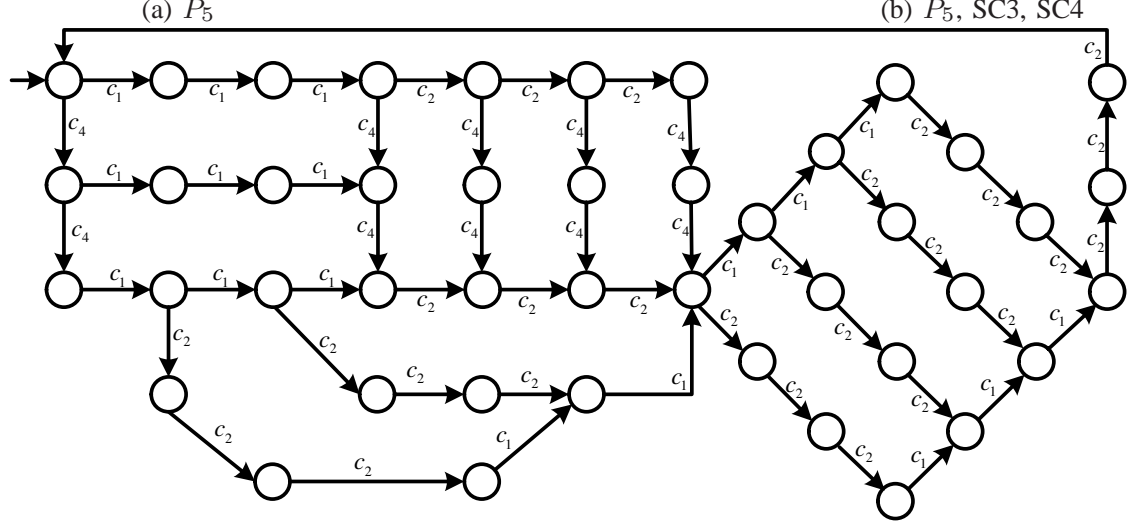

(c) $P_{6}$

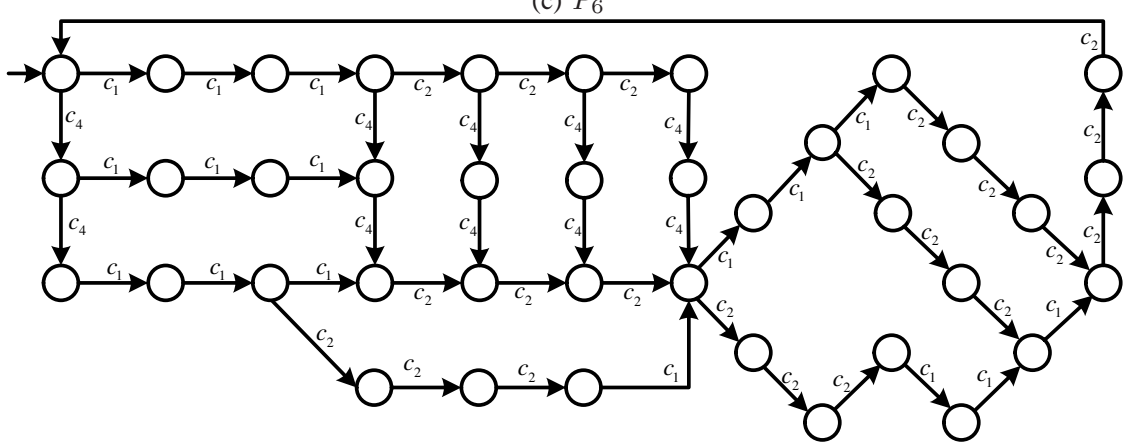

(d) $P_{6}, \mathrm{SC} 1$

Fig. 15: Scheduling map of $\mathbb{S}^{1}$.

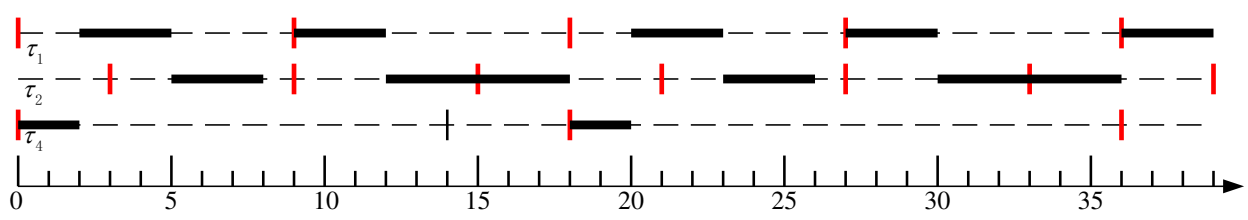

Fig. 16: PTS scheduling map of $\mathbb{S}^{2}$.

${ }_{853} \quad P_{1}=\left(\begin{array}{lllll}0 & 1 & 1 & 1 & 1 \\ 1 & 0 & 1 & 1 & 1 \\ 1 & 1 & 0 & 1 & 1 \\ 1 & 1 & 1 & 0 & 1 \\ 1 & 1 & 1 & 1 & 0\end{array}\right) \quad P_{2}=\left(\begin{array}{lllll}0 & 1 & 1 & 1 & 1 \\ 1 & 0 & 1 & 1 & 1 \\ 0 & 0 & 0 & 0 & 0 \\ 1 & 1 & 1 & 0 & 1 \\ 1 & 1 & 1 & 1 & 0\end{array}\right)$

$$
\begin{aligned}
& P_{5}=\left(\begin{array}{lllll}
0 & 0 & 1 & 0 & 0 \\
1 & 0 & 1 & 1 & 1 \\
1 & 1 & 0 & 1 & 1 \\
1 & 1 & 1 & 0 & 1 \\
1 & 1 & 1 & 1 & 0
\end{array}\right) P_{6}=\left(\begin{array}{lllll}
0 & 0 & 0 & 1 & 0 \\
1 & 0 & 1 & 1 & 1 \\
1 & 1 & 0 & 1 & 1 \\
1 & 1 & 1 & 0 & 1 \\
1 & 1 & 1 & 1 & 0
\end{array}\right) \\
& P_{7}=\left(\begin{array}{lllll}
0 & 0 & 0 & 0 & 1 \\
1 & 0 & 1 & 1 & 1 \\
1 & 1 & 0 & 1 & 1 \\
1 & 1 & 1 & 0 & 1 \\
1 & 1 & 1 & 1 & 0
\end{array}\right) P_{8}=\left(\begin{array}{lllll}
0 & 0 & 0 & 0 & 0 \\
0 & 0 & 0 & 0 & 0 \\
0 & 0 & 0 & 0 & 0 \\
0 & 0 & 0 & 0 & 0 \\
0 & 0 & 0 & 0 & 0
\end{array}\right)
\end{aligned}
$$


TABLE V: Uni-processor scheduler behaviors

\begin{tabular}{llll}
\hline$P$ & Super & RM & SM \\
\hline$P_{1}$ & $(15572,31333)$ & $(522,787)$ & $(2632,5921)$ \\
$P_{2}$ & $(13051,24466)$ & $(521,785)$ & $(2354,4364)$ \\
$P_{3}$ & $(6700,10480)$ & $(520,774)$ & $(1503,1935)$ \\
$P_{4}$ & $(13508,25868)$ & $(522,787)$ & $(2385,4602)$ \\
$P_{5}$ & $(13321,25536)$ & $(522,787)$ & $(2353,4538)$ \\
$P_{6}$ & $(13011,24428)$ & $(522,787)$ & $(2339,4326)$ \\
$P_{7}$ & $(13676,26076)$ & $(522,787)$ & $(2427,4649)$ \\
$P_{8}$ & $(5140,7396)$ & $(515,764)$ & $(1279,1528)$ \\
\hline
\end{tabular}

ing maps differ greatly. The preemption matrix $P_{1}$ (resp., $P_{8}$ ) represents that the RTS is under the preemptive (resp., nonpreemptive) real-time scheduling; and the supervisor provides the most flexible (resp., restricted) behaviors. The preemption matrix $P_{2}$ indicates that the real-time scheduling plan is that the execution of $\tau_{3}$ cannot be preempted by any other task. The preemption matrix $P_{3}$ represents that the execution of $\tau_{1}$ can be preempted by $\tau_{2}, \tau_{2}$ by $\tau_{3}, \tau_{3}$ by $\tau_{4}, \tau_{4}$ by $\tau_{5}$, and $\tau_{5}$ by $\tau_{1}$, respectively. Importantly, the release maps for $P_{4}$, $P_{5}, P_{6}$, and $P_{7}$, are isomorphic with the release map for $P_{1}$. However, the scheduling maps are differ substantially from one another. The reason is that $P_{4}, P_{5}, P_{6}$, and $P_{7}$ show that the execution of $\tau_{1}$ can only be preempted by $\tau_{2}, \tau_{3}, \tau_{4}$, and $\tau_{5}$, respectively.

\section{2) Multi-processor system:}

Suppose that the tasks are running in an RTS that possesses two processors and their corresponding preemption-matrices are identical. We need to calculate the processor behavior based on each processor individually. Suppose that tasks $\tau_{1}$ and $\tau_{2}$ are running in processor $\Xi_{1}$, and $\tau_{3}$ and $\tau_{4}$ are running in processor $\Xi_{2}$. Task $\tau_{5}$ is a newly arrived task and is allowed to enter any processor. By applying preemption matrix $P_{4}$ to both $\Xi_{1}$ and $\Xi_{2}$, the corresponding supremal supervisors, release maps, and scheduling maps are recorded in Table VI. If task $\tau_{5}$ enters $\Xi_{1}$ (resp., $\Xi_{2}$ ), the corresponding new system behavior is represented by $\Xi_{1}^{\prime}$ (resp., $\Xi_{2}^{\prime}$ ).

TABLE VI: Multi-processor scheduler behaviors of of $\mathbb{S}^{2}$

\begin{tabular}{llll}
\hline$\Xi$ & Super & RM & SM \\
\hline$\Xi_{1}$ & $(183,209)$ & $(20,21)$ & $(45,54)$ \\
$\Xi_{2}$ & $(87,101)$ & $(8,9)$ & $(18,22)$ \\
$\Xi_{1}^{\prime}$ & $(253,313)$ & $(25,26)$ & $(62,76)$ \\
$\Xi_{2}^{\prime}$ & $(344,424)$ & $(32,35)$ & $(76,98)$ \\
\hline
\end{tabular}

\section{E. Example. 3.}

Consider a manufacturing cell as an example. As shown in Fig. 18, a robot $R$ is utilized to transport two types of workpieces, W1 and W2, to a conveyor. Two pieces of W1 (resp., W2) are released to the input buffer $B_{1}$ (resp., $B_{2}$ ) simultaneously in every six (resp., three) seconds. The robot $R$ has capacity one; and transporting each piece takes one second. Thus, we define two tasks $\tau_{1}=\{0,2,6,6\}$ (resp., $\left.\tau_{2}=\{0,2,3,3\}\right)$ to represent the transportion of the two pieces of W1 (resp., W2) by $R$, respectively. Consequently, we have a system $\mathbb{S}=\left\{\tau_{1}, \tau_{2}\right\}$.

Suppose that the preemption matrix with respect to $\mathbb{S}$ is

$$
P_{1}=\left(\begin{array}{cc}
0 & 0 \\
1 & 0
\end{array}\right) .
$$

The scheduling can be considered as an FP scheduling, i.e., task $\tau_{1}$ cannot be preempted by $\tau_{2}$; and $\tau_{2}$ is allowed to be preempted by $\tau_{1}$. In other words, the robot must transport the two W1's in two adjacent seconds, but it is not necessary for transporting W2. By utilizing SCT, the supervisor calculated by supcon is represented by a DES with 21 states and 27 transitions. As shown in Fig. 19, the release (resp., scheduling) map is represented by a generator with three (resp., six) states and three (resp., six) transitions. As shown in Fig. 19(b), no preemption scheduling sequences are found. This requires that the robot $R$ must transport the workpieces in the following order periodically:

W2, W2, W1, W1, W2, W2.

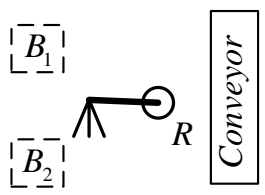

Fig. 18: Manufacturing cell.

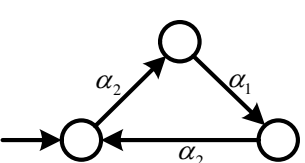

(a) Release map

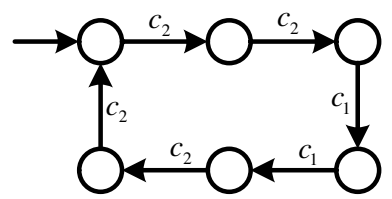

(b) Scheduling map
Fig. 19: FP scheduling.

Suppose that the preemption matrix is

$$
P_{2}=\left(\begin{array}{ll}
0 & 1 \\
1 & 0
\end{array}\right),
$$

i.e., the real-time scheduling is preemptive. This means that the robot can transport W1 and W2 in any order. By using SCT to calculate the supervisor, it is represented by a DES with 37 states and 55 transitions. As shown in Fig. 20, the release map (resp., scheduling map) is represented by a generator with four (resp., ten) states and five (resp., 14) transitions. The scheduling map in Fig. 20(b) provides nine safe execution sequences.

If we also require that, based on $P_{2}$, the WCRT of task $\tau_{1}$ be $\mathbb{W}_{1}=5$, i.e., the two $\mathrm{W} 1$ 's must be transported in the first five seconds after their release, then we obtain a supervisor that is represented by a DES with 63 states and 91 transitions. The release map is isomorphic with the map depicted in 20(a); and the scheduling map is displayed in Fig. 21. 


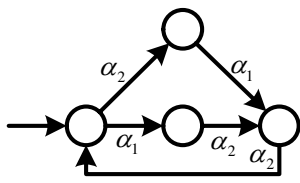

(a) Release map

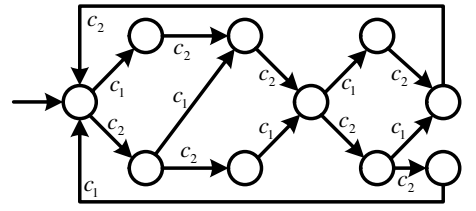

(b) Scheduling map

Fig. 20: Preemptive scheduling.

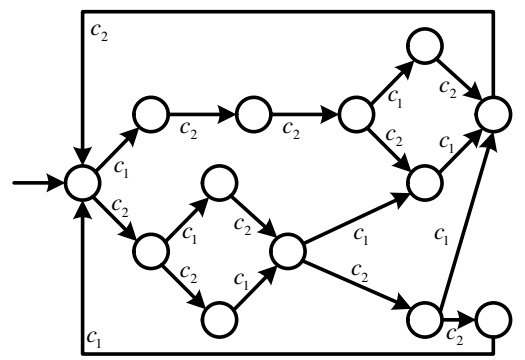

Fig. 21: Preemptive scheduling with $\mathbb{W}_{1}=5$.

\section{DISCUSSION}

\section{A. Computational Complexity and Calculation Speed up}

The real-time scheduling is based on the computation of the supremal controllable sublanguage with respect to a finite DES. According to [13] and [24], the computation of the supremal controllable sublanguage with respect to a finite DES can be completed in polynomial time. Similar to [13], the computational complexity of the presented method in this article is characterized by 1) the modeling of a processor time unit as a distinct event in the DES framework and 2) the exponential growth in the number of states when synchronous product is utilized to combine individual tasks into the plant. The computational complexity of the supremal sublanguage of a specification is $O\left(m^{2} n^{2}\right)$, where $m$ and $n$ are the sizes of the final state set of the plant $\mathbf{G}$ and the specification $\mathbf{S}$, respectively. Similar to [13], this remains a challenge in "scaling up" the proposed method for the real-time scheduling based on SCT. In the worst case, the synchronous product of two discrete-event systems, with $x$ and $y$ states, respectively, results in a more complex system with $x \cdot y$ states. A method to speed up the calculation is to reduce the number of states in the plant and specification. In order to achieve this goal, we divide the calculations into three steps. Each step considers different specifications as follows.

- Step 1: nonblocking and matrix-based conditionalpreemption specifications,

- Step 2: nonblocking and WCET-based conditionalpreemption specifications, and

- Step 3: nonblocking and WCRT-based conditionalpreemption specifications.

As the commutative diagram shown in Fig. 22, Algorithm 1 is revised to be Algorithm 2, in which NS, PS, CS, and RS represent nonblocking specifications, matrix-based conditional-preemption specifications, WCETbased conditional-preemption specifications, and WCRT-based conditional-preemption specifications, respectively.

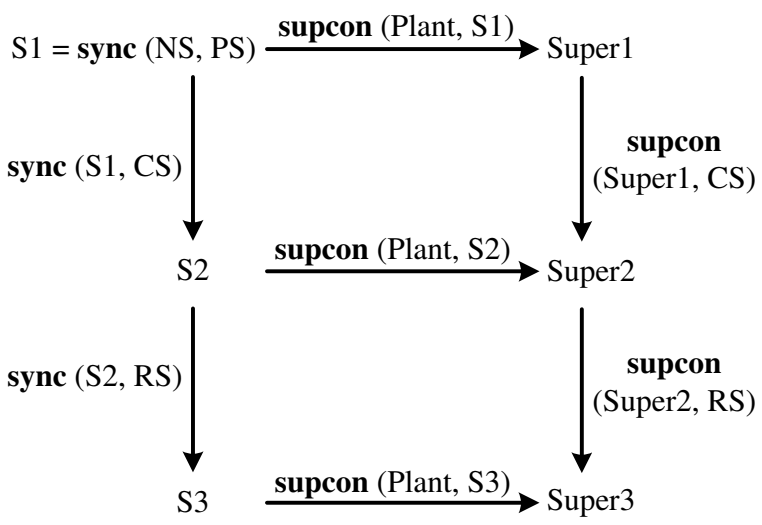

Fig. 22: Commutative diagram.

\begin{tabular}{l}
\hline Algorithm 2 Supervisory control of RTS \\
\hline 1. Build Plant by sync; \\
2. Build S1, S2, and S3 by sync; \\
3. Super1 = supcon (Plant, S1); \\
4. Super2 = supcon (Super1, S2); \\
5. Super3 = supcon (Super2, S3). \\
\hline
\end{tabular}

For example, the scheduling of $\mathbb{S}^{1}$ based on $P_{3}$ and SC1 can be calculated in the first two steps as listed in Table VII. Moreover, if we require that the WCRT of task $\tau_{1}$ is $\mathbb{W}_{1}=4$, only one such sequence exists, which is shown in Fig. 23. Accordingly, $\mathbb{S}^{1}$ can be scheduled in order $\tau_{1} \tau_{2} \tau_{1} \tau_{2} \tau_{2}$.

TABLE VII: Uni-processor scheduler behaviors of $\mathbb{S}^{1}$

\begin{tabular}{lllll}
\hline Plant & Spec & Super & RM & SM \\
\hline SYS1 & NS & Super1 $(69,87)$ & $(6,7)$ & $(24,27)$ \\
Super1 & P3 & Super2 $(65,82)$ & $(6,7)$ & $(22,24)$ \\
Super2 & SC1 & Super3 $(45,52)$ & $(5,5)$ & $(15,15)$ \\
\hline
\end{tabular}

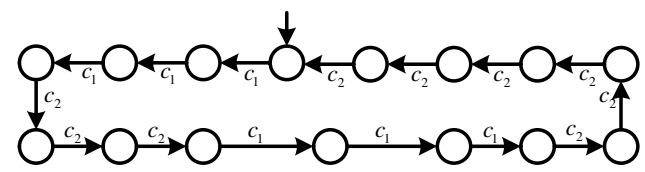

Fig. 23: Scheduling map of $\mathbb{S}^{1}$ with $P_{3}$, SC1, and SR1.

\section{B. Comparison with the CW Model}

In [13], a TDES model is proposed to represent periodic real-time tasks. A task is represented by a TDES

$$
\mathbf{G}_{i}=\left(Q_{i}, \Sigma_{i}, \delta_{i}, q_{0 i}, Q_{m i}\right)
$$

where $\Sigma_{i}$ consists of

- $t$ : tick event,

- $\gamma_{i}$ : the release event of $\tau_{i}$,

- $\alpha_{i}$ : the execution of $\tau_{i}$ is started, and

- $\beta_{i}$ : the execution of $\tau_{i}$ is finished.

Suppose that after being enabled, events $\gamma_{i}, \alpha_{i}$, and $\beta_{i}$ should wait for $t_{\gamma_{i}}, t_{\alpha_{i}}$, and $t_{\beta_{i}}$ ticks, respectively, until they are eligible to occur. Formally, the timer bounds are
963 964 965 966 967 


$$
\begin{aligned}
\text { - } \gamma_{i}:=\left\{\begin{array}{c}
{[0,0],} \\
\text { if } \tau_{i} \text { releases at } r_{1,1} \\
{\left[T_{i}-t_{\alpha_{i}}-t_{\beta_{i}}, T_{i}-t_{\alpha_{i}}-t_{\beta_{i}}\right],} \\
\text { if }(\forall j>1) \tau_{i} \text { releases at } r_{i, j}
\end{array}\right. \\
\text { - } \alpha_{i}:=\left[0, D_{i}-t_{\beta_{i}}\right], \text { and } \\
\text { - } \beta_{i}:=\left[t_{\beta_{i}}, t_{\beta_{i}}\right] .
\end{aligned}
$$

In the $\mathrm{CW}$ model [13], $t_{\beta_{i}}=C_{i}$. The regular languages utilized to describe the tasks represented by TDES/DES are different. In comparison, in the TDES (resp. DES) model, the processor execution of task $\tau_{i}$ is represented by $t$ (resp. $c_{i}$ ). Consequently, in the TDES (resp. DES) model, the execution of different tasks is considered as the same (resp. different) events. Hence, in the synchronous product, they will occur simultaneously (resp. separately). For preemptive real-time scheduling, becasue of the nature of the real-time scheduling, the execution of two or more tasks within a uni-processor cannot happen simultaneously. Thus, the CW TDES model cannot be utilized to schedule real-time tasks conditionallypreemptively.

\section{Remark:}

Suppose that we have two substrings $s_{1}=c_{1} c_{1}$ and $s_{2}=c_{2}$ under condition that exists $w_{1}, v_{1} \in \Sigma_{1}^{*}, w_{1} s_{1} v_{1} \in L\left(\mathbf{G}_{1}\right)$ and exists $w_{2}, v_{2} \in \Sigma_{2}^{*}, w_{2} s_{2} v_{2} \in L\left(\mathbf{G}_{2}\right)$. The execution of $s_{1}$ and $s_{2}$ within a uni-processor needs to take three time units. Let $L_{1}=\left\{s_{1}\right\} \subset \Sigma_{1}^{\prime *}$ and $L_{2}=\left\{s_{2}\right\} \subset \Sigma_{2}^{\prime *}$ with $\Sigma_{1}^{\prime}=\left\{c_{1}\right\} \subset$ $\Sigma_{1}$ and $\Sigma_{2}^{\prime}=\left\{c_{2}\right\} \subset \Sigma_{2}$, respectively. Their synchronous product is $L_{1} \| L_{2}=\left\{c_{2} c_{1} c_{1}, c_{1} c_{2} c_{1}, c_{1} c_{1} c_{2}\right\}$; it represents all the possible executions correctly. Similarly, according to the TDES model presented in [13], $c_{1}$ and $c_{2}$ are both replaced by the tick event $t$. Thus, we can obtain $s_{1}=t t, s_{2}=t$, $L_{1}=\left\{s_{1}\right\} \subset \Sigma_{1}^{\prime *}, L_{2}=\left\{s_{2}\right\} \subset \Sigma_{2}^{\prime *}, \Sigma_{1}^{\prime}=\{t\} \subset \Sigma_{1}$, and $\Sigma_{2}^{\prime}=\{t\} \subset \Sigma_{2}$. The synchronous product of $L_{1}$ and $L_{2}$ is $L_{1} \| L_{2}=\emptyset$. Evidently, this model cannot actually represent real-time scheduling.

\section{Comparison with (Time) Petri Nets Models}

Recently, preemptive time Petri nets (pTPN) are developed in [25], [26], and [27] to dynamically schedule real-time systems. These studies have significant improvements in realtime scheduling based on time Petri nets. However, the realtime scheduling in [25], [26], and [27] can only be applied to the FP real-time scheduling. To the best of our knowledge, conditional-preemption is not addressed in these studies. For example, by using the models proposed in [25], [26], and [27], no fixed priority can be assigned to the tasks running in the motivating example of the present paper. Consequently, the real-time scheduling based on SCT is more general than the pTPN model.

Real-time scheduling can also be modeled by Petri nets (PN) with time constraints [28], [29]. However, the proposed approach in [28] faces the schedulability conditions of the offline scheduling problems; and finding the optimal offline periodic schedule is a challenge. The problems of [28] are addressed in [29]. However, it is arguable whether or not that a PN with time constraints is a sufficiently general model to schedule real-time systems. Furthermore, not all the conditional specifications considered in the present paper are addressed in the PN model with time constraints.

In comparison, in this study, by utilizing SCT offline, all the safe execution sequences can be found if any exist. Otherwise, the supervisor of the system is empty. On the basis, we conclude that the developed conditionally-preemptive scheduling algorithm is more general than the scheduling based on (time) Petri nets models.

\section{CONClusion}

This study reports a formal constructive method for realtime periodic tasks via a DES model. For both uni-processor and multi-processor systems, the behavior of a processor can be established by the synchronous product of the DES models of all tasks running in it. The tasks can be scheduled without considering their priorities. This paper presents two sets of conditional-preemption specifications, i.e., matrixbased conditional-preemptions, and WCET-based conditionalpreemptions. Moreover, in order to control the system to be nonblocking and also limit the WCRT of the tasks, two corresponding sets of specifications are developed. The formal SCT of DES can be considered as a rigorous analysis and synthesis tool to schedule the RTS satisfying the hard deadlines. The procedure sync in TCT is utilized to generate the plant and global specifications. By utilizing the procedure supcon, all the conditionally-preemptive safe execution sequences can be calculated. These sequences can provide more choices than the EDF, PTS, DPS scheduling algorithms and the TDES SCTbased real-time scheduling proposed in [13]. In order to speed up the calculation, a commutative diagram is proposed to calculate the supervisors in three steps. The off-line scheduling algorithm presented in this study can be applied in a practical context to schedule the real-world uni-processor and multiprocessor systems. In future work, we will focus on the realtime conditionally-preemptive scheduling of sporadic tasks.

\section{REFERENCES}

[1] L. Sha, T. Abdelzaher, K. E. Årzén, A. Cervin, T. Baker, A. Burns, G. Buttazzo, M. Caccamo, J. Lehoczky, and A. K. Mok, "Real time scheduling theory: A historical perspective," Real-time systems, vol. 28, no. 2, pp. 101-155, 2004.

[2] R. I. Davis, "A review of fixed priority and EDF scheduling for hard real-time uniprocessor systems," ACM SIGBED Review, vol. 11, no.1, pp. 8-19, 2014.

[3] C. L. Liu and J. W. Layland, "Scheduling algorithms for multiprogramming in a hard real time environment," J. Assoc. Comput. Mach., vol. 20, no. 1, pp. 46-61, Jan. 1973.

[4] M. S. Fineberg, and O. Serlin, "Multiprogramming for hybrid computation," in Proc. AFIPS Fall Joint Computing Conference, pp. 1-13, 1967.

[5] J. Y. T. Leung, and J. Whitehead, "On the complexity of fixedpriority scheduling of periodic real-time tasks, "Performance Evaluation (Netherlands), vol. 2, no. 4, pp. 237-250, 1982

[6] A. K. Mok, "Fundamental design problems of distributed systems for the hard-real-time environment," Ph.D. Thesis, Department of Electrical Engineering and Computer Science, Massachusetts Institute of Technology, Cambridge, Massachusetts, 1983.

[7] M. L. Dertouzos, "Control robotics: the procedural control of physical processes," in Proc. IFIP cong., pp. 807-813, 1974.

[8] S. Baruah, R. Howell, and L. Rosier, "Algorithms and complexity concerning the preemptive scheduling of periodic real-time tasks on one processor," Real-Time Syst., vol. 2, pp. 301-324, 1990.

[9] R. R. Howell, M. K. Venkatrao, "On non-preemptive scheduling of recurring tasks using inserted idle time," Inform. Comput. J., vol. 117, no. 1, pp. 50-62, 1995. 
[10] J. J. Li, L. C, Shu, J. J. Chen, and G. H. Li, "Energy-efficient scheduling in nonpreemptive systems with real-time constraints," IEEE Trans. Syst. Man Cybern.: Syst., vol. 43, no. 2, pp. 332-344, 2013.

[11] Y. N. Xia, M. C. Zhou, X. Luo, S. C. Pang, and Q. S. Zhu, "A stochastic approach to analysis of energy-aware DVS-enabled cloud datacenters," IEEE Trans. Syst. Man Cybern.: Syst., vol. 45, no. 1, pp. 73-83, 2015.

12] D. Li, M. Li, X. Meng, and Y. Tian, "A hyperheuristic approach for intercell scheduling with single processing machines and batch processing machines," IEEE Trans. Syst. Man Cybern.: Syst., vol. 45, no. 2, pp. $315-325,2015$

[13] P. C. Y. Chen and W. M. Wonham, "Real-time supervisory control of a processor for non-preemptive execution of periodic tasks," Real-Time Syst., vol. 23, pp. 183-208, 2002.

[14] W. M. Wonham, Supervisory control of discrete-event systems, Department of Electrical and Computer Engineering, University of Toronto, 2015. Available at http://www.control.utoronto.ca/DES.

[15] P. J. Ramadge and W. M. Wonham, "Supervisory control of a class of discrete event processes," SIAM J. Contr. Optim., vol. 25, no. 1, pp. 206-230, 1987.

[16] A. Dhananjayan, T. S. Kiam, "A metric temporal logic specification interface for real-time discrete-event control," IEEE Trans. Syst. Man Cybern.: Syst., vol. 44, no. 9, pp. 1204-1215, 2014.

17] J. Ye, Z. W. Li, and A. Giua, "Decentralized supervision of Petri nets with a coordinator," IEEE Trans. Syst. Man Cybern.: Syst., vol. 45, no. 6, pp. 955-966.

18] D. You, S. G. Wang, M. C. Zhou, "Synthesis of monitor-based livenessenforcing supervisors for $\mathrm{S}^{3} \mathrm{PR}$ With $\xi$-Resources," IEEE Trans. Syst. Man Cybern.: Syst., vol. 45, no. 6, pp. 967-975.

19] G. C. Buttazzo, M. Bertogna, and G. Yao, "Limited preemptive scheduling for real-time systems. A survey," IEEE Trans. Ind. Inform., vol. 9, no. 1 , pp. $3-15,2013$

[20] Y. Wang and M. Saksena, "Scheduling fixed-priority tasks with preemption threshold," in Proc. Proceedings of the Real-Time Computing Systems and Applications, pp. 328-335, 1999.

[21] S. Baruah, "The limited-preemption uniprocessor scheduling of sporadic task systems," Real-Time Syst, 2005. in Proc, 17th Euromicro Conf. Real-Time Syst., pp. 137-144, 2005.

[22] J. Y. T. Leung and M. L. Merrill, "A note on preemptive scheduling of periodic real-time tasks," Inform. Proc. Letters, vol. 11, no.3, pp. 115-1118, 1980

[23] F. Singhoff, J. Legrand, L. Nana, and L. Marce, "Cheddar: A flexible real time scheduling framework," in Proc. Int. ACM SIGAda Conf., pp. $1-8,2004$.

24] P. J. Ramadge and W. M. Wonham, "The control of discrete event systems," Proceedings of the IEEE, vol. 77, no. 1, pp. 81-98, 1989.

[25] L. Carnevali, L. Ridi, and E. Vicario, "Putting preemptive time Petri nets to work in a V-Model SW life cycle," IEEE Trans. Softw. Eng., vol. 37, no. 6, pp. 826-844, 2011.

[26] I. Bicchierai, G. Bucci, L. Carnevali, and E. Vicario, "Combining UMLMARTE and preemptive time Petri nets: an industrial case study," IEEE Trans. Ind. Inform., vol. 9, no.4, pp. 1806-1818, 2013.

27] L. Carnevali, A. Pinzuti, and E. Vicario, "Compositional verification for hierarchical scheduling of real-time systems," IEEE Trans. Softw. Eng., vol. 39, no. 5, pp. 638-657, 2013.

$28]$ N. Q. Wu and M. C. Zhou, "Modeling, analysis and control of dual-arm cluster tools with residency time constraint and activity time variation based on Petri nets," IEEE Trans. Autom. Sci. Eng., vol. 9, no. 2, pp. 446-454, 2012.

[29] Y. Qiao, N. Q. Wu, and M. C. Zhou, "Real-time scheduling of single-arm cluster tools subject to residency time constraints and bounded activity time variation," IEEE Trans. Autom. Sci. Eng., vol. 9, no. 3, pp. 564-577, 2012 .

\section{Appendix 1.}

Synthesis procedures in TCT [14]:

DES2 = allevents (DES1) is a marked one-state DES selflooped with all the events of DES1.

DES = create (DES) is a new discrete-event system (DES). Option 1 allows fast user input via a sequence of prompts, resulting in direct creation of a .DES file. Option 2 allows the user to create a text (.ATS) file with any ASCII text editor; this file can be converted to a .DES file using the TCT procedure FD.

True/False = isomorph (DES1, DES2) tests whether DES1 and DES2 are identical up to renumbering of states (but with initial state held fixed at 0); if so, their state correspondence is displayed.
DES2 $=$ project $($ DES1, [NULL/IMAGE EVENTS]) is a generator of the projected closed and marked languages of DES1, under the natural projection specified by the listed Null or Image events. In decentralized control, DES2 could be an observer's local model of DES1.

DES2 = relabel $($ DES1, [OLD-NEW EVENT LABEL PAIRS] $)$ coincides with DES1, except for a mapping of specified event labels in DES1; unmapped labels are unchanged. Not for use with vocalized DES.

DES3 $=$ supcon $(D E S 1$, DES2) is a trim generator for the supremal controllable sublanguage of the marked legal language generated by DES2 with respect to the plant DES1. DES3 provides a proper supervisor for DES1.

DES3 = sync (DES1, DES2) is the (reachable) synchronous product of DES1 and DES2.

\section{Appendix 2.}

Corresponding TCT MAKEIT file for TASK3 and TASK4:

TASK 2 = create $($ TASK $2,[\operatorname{mark} 0,1,2,3,4,9,10,15,16,21$, 22], $[\operatorname{tran}[0,0,1],[0,19,1],[0,39,1],[0,49,1],[1,0,2],[1,19$, 2], [1, 39, 2], [1, 49, 2], [2, 0,3], [2, 19, 3], [2, 39, 3], [2, 49, 3], [3, $20,4],[4,19,10],[4,21,5],[4,39,10],[4,49,10],[5,19,11],[5$, $29,6],[5,39,11],[5,49,11],[6,19,12],[6,29,7],[6,39,12],[6$, $49,12],[7,19,13],[7,29,8],[7,39,13],[7,49,13],[8,22,9],[9$, $0,15],[9,19,15],[9,39,15],[9,49,15],[10,19,16],[10,21,11]$, $[10,39,16],[10,49,16],[11,19,17],[11,29,12],[11,39,17],[11$, 49, 17], [12, 19, 18], [12, 29, 13], [12, 39, 18], [12, 49, 18], [13, 19, 19], [13, 29, 14], [13, 39, 19], [13, 49, 19], [14, 22, 15], [15, 0, 21], $[15,19,21],[15,39,21],[15,49,21],[16,19,22],[16,21,17],[16$, $39,22],[16,49,22],[17,19,23],[17,29,18],[17,39,23],[17,49$, 23], [18, 19, 24], [18, 29, 19], [18, 39, 24], [18, 49, 24], [19, 19, 25], [19, 29, 20], [19, 39, 25], [19, 49, 25], [20, 22, 21], [21, 0, 3], $[21,19,3],[21,39,3],[21,49,3],[22,21,23],[23,29,24],[24$, $29,25],[25,29,26],[26,22,3]])(28,81)$

\section{Appendix 3.}

The generated files for the specifications are recorded.

1. Nonblocking specifications:

SN1 $=$ allevents $($ TASK1 $)(1,8)$

SN2 $=$ allevents $($ TASK2 $)(1,8)$

SN3 $=$ allevents $($ TASK3 $)(1,8)$

SN4 = allevents $($ TASK4 $)(1,8)$

2. Matrix-based conditional-preemption specifications:

$\mathrm{SP} 1=$ create $(\mathrm{SP} 1,[\operatorname{mark} 0],[\operatorname{tran}[0,0,0],[0,11,1],[0,29,0]$, $[0,39,0],[0,49,0],[1,12,0],[1,19,1]])(2,7)$

$\mathrm{SP} 2=$ create $(\mathrm{SP} 2,[\operatorname{mark} 0],[\operatorname{tran}[0,0,0],[0,19,0],[0,39,0]$, $[0,49,0],[0,21,1],[1,22,0],[1,29,1]])(2,7)$

SP4 = create $(S P 4,[\operatorname{mark} 0],[\operatorname{tran}[0,0,0],[0,19,0],[0,29,0]$,

$[0,39,0],[0,41,1],[1,42,0],[1,49,1]])(2,7)$

$\mathrm{SP} 12=$ create $(\mathrm{SP} 12,[\operatorname{mark} 0],[\operatorname{tran}[0,0,0],[0,11,1],[0,29$, $0],[0,39,0],[0,49,0],[1,12,0],[1,19,1],[1,29,1]])(2,8)$ SP24 = create $(S P 24,[$ mark 0], $[\operatorname{tran}[0,0,0],[0,19,0],[0,21$, 1], [0, 39, 0], [0, 49, 0], [1, 22, 0], [1, 29, 1], [1, 49, 1]]) $(2,8)$ SP41 = create $(S P 41,[$ mark 0], $[\operatorname{tran}[0,0,0],[0,19,0],[0,29$, 0], [0, 39, 0], [0, 41, 1], [1, 19, 1], [1, 42,0], [1, 49, 1]]) $(2,8)$ SP134 = create $(\mathrm{SP} 134,[\operatorname{mark} 0],[\operatorname{tran}[0,0,0],[0,11,1],[0$, $29,0],[0,39,0],[0,49,0],[1,12,0],[1,19,1],[1,39,1],[1,49$, 1]]) $(2,9)$

3. WCET-based conditional-preemption specifications:

$\mathrm{SC} 1=$ create $(\mathrm{SC} 1,[\operatorname{mark} 0],[\operatorname{tran}[0,0,0],[0,11,1],[0,29,0]$, $[0,39,0],[0,49,0],[1,19,2],[1,29,1],[1,39,1],[1,49,1],[2$, $19,3],[2,39,2],[2,49,2],[3,12,0],[3,19,3],[3,29,3],[3,39$, $3],[3,49,3]])(4,17)$

SC2 $=$ create $(\mathrm{SC} 2,[\operatorname{mark} 0],[\operatorname{tran}[0,0,0],[0,19,0],[0,21,1]$, $[0,39,0],[0,49,0],[1,19,1],[1,29,2],[1,39,1],[1,49,1],[2$, $19,2],[2,29,3],[2,39,2],[2,49,2],[3,29,4],[3,39,3],[3,49$, $3],[4,19,4],[4,22,0],[4,39,4],[4,49,4]])(5,20)$

$\mathrm{SC} 3=$ create $(\mathrm{SC} 1,[\operatorname{mark} 0],[\operatorname{tran}[0,0,0],[0,11,1],[0,29,0]$, $[0,39,0],[0,49,0],[1,19,2],[1,29,1],[1,39,1],[1,49,1],[2$, 
$19,3],[2,29,2],[2,39,2],[3,12,0],[3,19,3],[3,29,3],[3,39$, $3],[3,49,3]])(4,17)$

SC4 = create $(\mathrm{SC} 2,[\operatorname{mark} 0],[\operatorname{tran}[0,0,0],[0,19,0],[0,21,1]$, $[0,39,0],[0,49,0],[1,19,1],[1,29,2],[1,39,1],[1,49,1],[2$, $19,2],[2,29,3],[2,39,2],[2,49,2],[3,29,4],[3,19,3],[3,39$, $3],[4,19,4],[4,22,0],[4,39,4],[4,49,4]])(5,20)$

\section{WCRT-based conditional-preemption specifications:}

$\mathrm{SR} 1=$ create $(\mathrm{SR} 1,[\operatorname{mark} 0],[\operatorname{tran}[0,0,0],[0,10,1],[0,29$, $0],[0,39,0],[0,49,0],[1,19,2],[1,29,2],[1,39,2],[1,49,2]$, $[2,12,0],[2,19,3],[2,29,3],[2,39,3],[2,49,3],[3,12,0],[3$, $19,4],[3,29,4],[3,39,4],[3,49,4],[4,12,0],[4,19,5],[4,29$, $5],[4,39,5],[4,49,5],[5,12,0]])(6,25)$

SR4 $=$ create $(S R 4,[$ mark 0], [tran $[0,0,0],[0,19,0],[0,29,0]$, $[0,39,0],[0,40,1],[1,19,2],[1,29,2],[1,39,2],[1,49,2],[2$, $19,3],[2,29,3],[2,39,3],[2,42,0],[3,42,0]])(4,15)$ 